Mathematics of Control, Signals, and Systems manuscript No. (will be inserted by the editor)

\title{
Local nested transverse feedback linearization
}

\author{
Alireza Doosthoseini · Christopher \\ Nielsen
}

Received: date / Accepted: date

\begin{abstract}
We study two local feedback equivalence problems for a nonlinear control-affine system with two nested, controlled invariant, embedded submanifolds in its state space. The first, less restrictive, result gives necessary and sufficient conditions for the dynamics of the system restricted to the larger submanifold and transversal to the smaller submanifold to be linear and controllable. This normal form facilitates designing controllers that locally stabilize the smaller set relative to the larger set. The second, more restrictive, result additionally imposes that the transversal dynamics to the larger set be linear and controllable. This result can simplify designing controllers to locally stabilize the larger submanifold. This is illustrated by sufficient conditions under which these normal forms can be used to locally solve a nested set stabilization problem.
\end{abstract}

Keywords Feedback equivalence · partial feedback linearization · nested sets $\cdot$ nonlinear control systems.

This research was partially supported by the Natural Sciences and Engineering Research Council of Canada (N.S.E.R.C.).

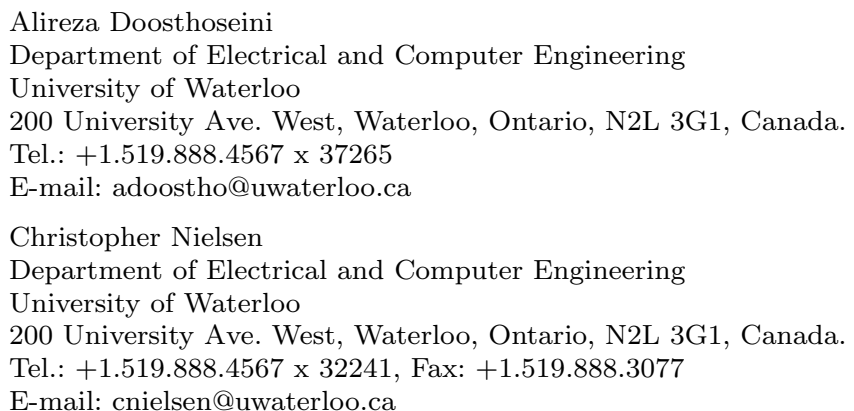

E-mail: cnielsen@uwaterloo.ca 


\section{Introduction}

Feedback equivalence problems have been extensively studied in the last thirty five years and their solutions have been a valuable tool in making many nonlinear control design problems tractable. Two control systems are said to be feedback equivalent if there exists a coordinate transformation and a local feedback transformation mapping trajectories of two control systems to each other. A large portion of the control literature on feedback equivalence is dedicated to finite-dimensional, autonomous, deterministic, nonlinear control-affine systems and the feedback equivalence is local, i.e., valid in a neighbourhood of a point in the system's state space. Most of the studies on this field are heavily influenced by the seminal works of Poincaré [32] and Cartan [4]. Poincaré found sufficient conditions for a dynamic system to be locally equivalent to a linear one by means of an analytic transformation. In Cartan's method of equivalence a Pfaffian system generated by differential forms is associated to a dynamical system. The feedback equivalence problem is analyzed by studying the feedback equivalence of the corresponding Pfaffian systems. Ever since Cartan's method of equivalence many studies have concentrated on this topic the most prominent of which are $[11,12,13,14,34]$. A profound survey of different studies on feedback equivalence problem can be found in [33] and references therein.

A distinguished subdivision of studies on feedback equivalence is called feedback linearization in which feedback equivalence of a nonlinear control system to a controllable linear, or partially linear, system is sought. The motivation for studying this special case is clear. Rather than designing a feedback controller for the nonlinear control system, a potentially difficult task, the designer first finds the feedback equivalent linear system. The feedback law is designed for the feedback equivalent linear system using the rich set of design tools for this class of system and is subsequently implemented on the original nonlinear plant. Feedback equivalence to a linear control system, known as state-space exact feedback linearization, was initially raised in [20]. In [3] this problem was solved for single-input single-output nonlinear control systems. Extension to the multi-input multi-output nonlinear control systems was investigated in [35, 36]. In [17] the feedback equivalence of a control system to a partially linear control system, known as partial feedback linearization, was raised. In [23] partial feedback linearization yielding a linear subsystem of maximal size is investigated for single-input single-output systems. In [27, 28] the multi-input multi-output case is considered.

Frequently, the input of a control system is employed to control its output. Thus, a natural feedback equivalence problem is to find, if possible, a coordinate and feedback transformation linearizing the input-output dynamics. This problem is referred to as input-output feedback linearization and in $[17,18]$ is investigated for single-input single-output control systems. Similar results for multi-input multi-output control systems are discussed in [16]. The problems of input-output feedback linearization, partial feedback linearization and state-space exact feedback linearization are closely related. Specifically, the 
state-space exact linearization problem is solvable if and only if there exists an output function yielding full relative degree, while the partial feedback linearization problem is solvable if and only if there exists an output function yielding some well-defined vector relative degree.

Since the class of feedback linearizable systems is relatively small, the concept of approximate feedback linearization was first raised by [21] and later different aspects of which was studied in [24, 25, 22, 19].

Many feedback control specifications can be cast as the problem of stabilizing an appropriate set in a system's state space. For instance, the consensus problem [31] and the synchronization of oscillators [7] require that a subset of states in a collection of control systems converge to each other. These can be viewed as set stabilization problems. Among the different control design techniques developed for set stabilization, feedback equivalence to partially linear controllable systems is an important one. In [1] a method called transverse feedback linearization (TFL) is developed for stabilizing an open-loop orbit in the state space of a single-input single-output control system. TFL is a partial feedback linearization method with the extra requirement that the controllable linear subsystem represent the transversal dynamics to the orbit. In [30] TFL was generalized to multi-input multi-output control-affine systems and the target set is an arbitrary controlled-invariant embedded submanifold of the state-space.

While many control problems are cast as a single set stabilization problem, some can be formulated as a nested set stabilization problem. For instance, in [10] a problem called hierarchical control design is cast as a nested set stabilization in which a chain of closed nested sets $S_{1} \supset S_{2} \supset \cdots \supset S_{n}$ are required to be stabilized simultaneously. There, each set $S_{i}$ represents a set of control specifications and control specifications $i+1$ are met only if control specifications $i$ are met. Studies [5] and [6] have adopted the hierarchical viewpoint to solve the path following problem of the planar vertical take-off and landing aircraft and a coordinated path following problem for dynamic unicycles, respectively. Back-stepping method in [10] and passivity based method in [8] are employed to solve instances of nested set stabilization problems.

The main idea of this paper is to employ the feedback linearization method to facilitate a solution to an instance of a nested set stabilization problem in which we only consider two sets and assume the sets to be controlledinvariant embedded submanifolds. The generalization to more than two sets is straightforward. The approach taken in this paper is motivated by the results in [30]. More specifically, given a smooth, time-invariant, nonlinear controlaffine system and two nested, controlled-invariant, embedded submanifolds $S_{1}$ and $S_{2}$ the main problem in this paper is: to find a local diffeomorphism and feedback transformation, valid in a neighbourhood of a point on $S_{2}$, that bring the control system into a normal form whose dynamics restricted to $S_{1}$ and transversal to $S_{2}$ are linear and controllable. This problem, Problem 1 of the paper, is called partial local nested transversed feedback linearization problem. The solution of this problem simplifies designing controller for stabilization 
of $S_{2}$ relative to $S_{1}$ locally. We propose geometric necessary and sufficient conditions under which Problem 1 is solvable.

The normal form of Problem 1 does not necessarily simplify designing controllers that locally stabilize $S_{1}$. This is because, even if Problem 1 is solved, one generally needs a nonlinear controller to locally stabilize $S_{1}$. In particular, should one be interested in utilizing feedback linearization to locally stabilize $S_{1}$, we provide additional conditions under which the normal form of the first problem is refined and the dynamics transversal to $S_{1}$ are also linear and controllable. This normal form is simply called the local nested transverse feedback linearization normal form and it allows one to design the controllers for the local stabilization of $S_{1}$ and local stabilization of $S_{2}$ relative to $S_{1}$ separately. An issue that arises when stabilizing sets is that rendering $S_{1}$ asymptotically stable and $S_{2}$ asymptotically stable relative to $S_{1}$ does not imply that $S_{2}$ is asymptotically stable [8]. In this paper we provide sufficient conditions addressing this stability concern when using feedback equivalence to solve nested set stabilization problems.

The paper is organized as follows. In Section 2 the main problem, Problem 1, is formulated. In Section 3 we present preliminary results including the solution to a feedback equivalence problem concerning the restriction of control-affine system to nested controlled invariant submanifolds. In Section 4 we present the solution to Problem 1 in Theorem 2. In Section 5 the extension of Problem 1 is investigated. In Section 6 a nested set stabilization problem is introduced and the normal forms of Problems 1 and 3 are employed to design feedback control to solve it. Simulations are presented to illustrate the proposed method.

\subsection{Notation}

If $k$ is a natural number, $\mathbf{k}:=\{0, \ldots, k\}$. Let $U$ be an open subset of $\mathbb{R}^{n}$ and denote by $\operatorname{Diff}(U)$ the collection of diffeomorphisms with domain $U$. If $F: M \rightarrow N$ is a map between manifolds then $\mathrm{d} F_{x}: T_{x} M \rightarrow T_{F(x)} N$ denotes its differential. If $M$ and $N$ are vector spaces, then $\mathrm{d} F_{x}$ denotes the Jacobian matrix of $F$ at $x$. If $F: M \rightarrow N$ is a diffeomorphism between two manifolds, and if $v$ is a vector field on $M$, then the differential of $F$ can be used to define a vector field on $N$ by means of the push-forward map $F_{\star}$, defined as $F_{\star} v(q)=\left.\left(d F_{p} v(p)\right)\right|_{p=F^{-1}(q)}$. If $D$ is a non-singular distribution on a manifold $M, D^{\perp}$ is the orthogonal complement of $D$ obtained from the orthogonal structure on the tangent bundle $T M$. The non-singular distribution $D^{\perp}$ is a subbundle of $T M$ and satisfies, for each $p \in M, T_{p} M=D(p) \oplus D^{\perp}(p)$. Let $\operatorname{inv}(D)$ denote the involutive closure of $D$. We denote by $I_{m}$ the $m \times m$ identity matrix while $0_{m \times n}$ denotes an $m \times n$ matrix of zeros. Let $\mathrm{GL}(n, \mathbb{R})$ denote the group of non-singular $n \times n$ matrices with real entires. 


\section{Partial local nested transversal feedback linearization}

Consider a control-affine system

$$
\dot{x}=f(x)+\sum_{i=1}^{m} g_{i}(x) u_{i}=: f(x)+g(x) u
$$

where $x \in \mathbb{R}^{n}$ denotes the state, $u=\left(u_{1}, \cdots, u_{m}\right) \in \mathbb{R}^{m}$ is the control input, and $f: \mathbb{R}^{n} \rightarrow \mathbb{R}^{n}$ and $g_{i}: \mathbb{R}^{n} \rightarrow \mathbb{R}^{n}, i \in\{1, \cdots, m\}$, are smooth. To (1) we associate the family of distributions

$$
G_{i}:=\operatorname{span}\left\{a d_{f}^{j} g_{k}: 0 \leq j \leq i, 1 \leq k \leq m\right\}
$$

The vectors $g_{1}(x), \ldots, g_{m}(x)$ are assumed to be linearly independent at each $x \in \mathbb{R}^{n}$, i.e., $\operatorname{dim}\left(G_{0}(x)\right)=m$.

Definition 1 A closed connected submanifold $S \subseteq \mathbb{R}^{n}$ is controlled-invariant for (1) if there exists a smooth feedback $\bar{u}: S \rightarrow \mathbb{R}^{m}$ such that $S$ is invariant for the closed-loop system $\dot{x}=f(x)+g(x) \bar{u}(x)$.

Along with (1), we are also given two embedded submanifolds $S_{1} \subset \mathbb{R}^{n}$ and $S_{2} \subset \mathbb{R}^{n}$ with $s_{1}:=\operatorname{dim}\left(S_{1}\right), s_{2}:=\operatorname{dim}\left(S_{2}\right)$. The following assumption is made throughout this paper.

Assumption 1. The sets $S_{1}$ and $S_{2}$ are controlled-invariant embedded submanifolds for (1) and $S_{1} \supset S_{2}$.

The main problem investigated in this paper, Problem 1, seeks a decomposition of (1) into three subsystems modelling its evolution on (i) $S_{2}$ (ii) $S_{1} \backslash S_{2}$ and (iii) $\mathbb{R}^{n} \backslash S_{1}$, with the essential requirement that the dynamics on $S_{1} \backslash S_{2}$ be linear and controllable. Before formally stating the main problem, we review basic definitions.

Definition 2 Given an open set $U \subseteq \mathbb{R}^{n}$, a regular static feedback, denoted $(\alpha, \beta)$, on $U$ for control system (1) is a relation $u=\alpha(x)+\beta(x) v$ where $\alpha: U \rightarrow \mathbb{R}^{m}$ and $\beta: U \rightarrow \mathrm{GL}(m, \mathbb{R})$ are smooth mappings.

Definition 3 Two control systems, $\dot{x}=f+g u$ and $\dot{\hat{x}}=\hat{f}+\hat{g} \hat{u}$, are feedback equivalent on an open set $U \subseteq \mathbb{R}^{n}$ if there exist a regular static feedback $(\alpha, \beta)$ on $U$ and a map $\Xi \in \operatorname{Diff}(U)$ such that $\hat{f}=\Xi_{\star}(f+g \alpha)$ and $\hat{g}=\Xi_{\star}(g \beta)$.

Problem 1. (Partial local nested transversal feedback linearization problem). Given (1), nested sets $S_{1} \supset S_{2}$ satisfying Assumption 1 and a point $\bar{x} \in S_{2}$, find, if possible, a diffeomorphism $\Xi: U \rightarrow \Xi(U) \subset \mathbb{R}^{s_{2}} \times$ $\mathbb{R}^{s_{1}-s_{2}} \times \mathbb{R}^{n-s_{1}}, x \mapsto(\zeta, \mu, \xi)$, and a regular feedback transformation $(\alpha, \beta)$ valid in a neighbourhood $U \subseteq \mathbb{R}^{n}$ of $\bar{x}$, such that (1) is feedback equivalent to

$$
\begin{aligned}
& \dot{\zeta}=f_{1}(\zeta, \mu, \xi)+g_{11}(\zeta, \mu, \xi) v^{\|}+g_{12}(\zeta, \mu, \xi) v^{\|, \pitchfork}+g_{13}(\zeta, \mu, \xi) v^{\pitchfork} \\
& \dot{\mu}=A \mu+B v^{\|, \pitchfork}+f_{2}(\zeta, \mu, \xi)+g_{21}(\zeta, \mu, \xi) v^{\|}+g_{22}(\zeta, \mu, \xi) v^{\|, \pitchfork}+g_{23}(\zeta, \mu, \xi) v^{\pitchfork} \\
& \dot{\xi}=f_{3}(\zeta, \mu, \xi)+g_{31}(\zeta, \mu, \xi) v^{\|}+g_{32}(\zeta, \mu, \xi) v^{\|, \pitchfork}+g_{33}(\zeta, \mu, \xi) v^{\pitchfork}
\end{aligned}
$$


where

$$
\begin{gathered}
\Xi\left(S_{1} \cap U\right)=\{(\zeta, \mu, \xi) \in \Xi(U): \xi=0\}, \\
\Xi\left(S_{2} \cap U\right)=\{(\zeta, \mu, \xi) \in \Xi(U): \xi=0, \mu=0\},
\end{gathered}
$$

$f_{3}(\zeta, \mu, 0)=0, g_{31}(\zeta, \mu, 0)=0, g_{32}(\zeta, \mu, 0)=0, f_{2}(\zeta, \mu, 0)=0, g_{21}(\zeta, \mu, 0)=0$, $g_{22}(\zeta, \mu, 0)=0$, the pair $(A, B)$ is controllable, and $B$ is full rank.

Problem 1 seeks a coordinate and feedback transformation valid in a neighbourhood of $\bar{x}$ which generates a normal form with two types of decompositions. First the dynamics are decomposed into three subsystems; namely the $\xi-, \mu$-, and $\zeta$-subsystems. We call the $\xi$-subsystem the transversal dynamics to $S_{1}$. This is motivated by the fact that, in the light of (4a), stabilizing $S_{1} \cap U$ is equivalent, under mild assumptions, to stabilizing the $\xi$-subsystem. We call the $\mu$-subsystem the transversal dynamics of $S_{2}$, restricted to $S_{1}$. Similarly, this name is motivated by the fact that, in the light of (4b), stabilizing $S_{2} \cap U$ relative to $S_{1} \cap U$ is equivalent, under mild assumptions, to stabilizing the $\mu$-subsystem when $\xi=0$. The $\zeta$-subsystem is called the tangential dynamics to $S_{2}$ because when $\xi=0$ and $\mu=0$, the $\zeta$ dynamics govern the system's evolution on $\Xi\left(S_{2} \cap U\right)$.

The second type of decomposition is in the original $m$ inputs. They are partitioned into three groups : $v^{\|}, v^{\|, \pitchfork}$, and $v^{\pitchfork}$. The restrictions imposed on $f_{2}, f_{3}, g_{31}, g_{32}, g_{21}$, and $g_{22}$ after (4) imply that $\left(v^{\|}, v^{\|, \pitchfork}, v^{\pitchfork}\right)=(\star, \star, 0)$, where $\star$ represents arbitrary functions, renders $\Xi\left(S_{1} \cap U\right)$ locally invariant, i.e., $(\star, \star, 0)$ is a friend of $\Xi\left(S_{1} \cap U\right)$. Substituting $\xi=0$ and $v^{\pitchfork}=0$ in (3) the dynamics of (1) restricted to $\Xi\left(S_{1} \cap U\right)$ are

$$
\begin{aligned}
& \dot{\zeta}=f_{1}(\zeta, \mu, 0)+g_{11}(\zeta, \mu, 0) v^{\|}+g_{12}(\zeta, \mu, 0) v^{\|, \pitchfork} \\
& \dot{\mu}=A \mu+B v^{\|, \pitchfork} .
\end{aligned}
$$

The $\mu$-subsystem in (5) is linear and controllable and represents the dynamics of (1) restricted to $\Xi\left(S_{1} \cap U\right)$ and transversal to $\Xi\left(S_{2} \cap U\right)$. The control input $v^{\|, \pitchfork}$ can effectively be used to stabilize ${ }^{1} S_{2} \cap U$ relative to $S_{1} \cap U$. Finally $\Xi\left(S_{2} \cap U\right)$ is controlled-invariant with friend $\left(v^{\|}, v^{\|, \pitchfork}, v^{\pitchfork}\right)=(\star, 0,0)$. The dynamics of (1) restricted to $\Xi\left(S_{2} \cap U\right)$ are

$$
\dot{\zeta}=f_{1}(\zeta, 0,0)+g_{11}(\zeta, 0,0) v^{\|}
$$

Remark 1 In (3) the $\mu$-subsystem is not feedback linearized. It only becomes linear when it evolves on $\Xi\left(S_{1} \cap U\right)$. Thus (3) is less restrictive compared to a normal form in which the $\mu$-subsystem is linear off the set $\Xi\left(S_{1} \cap U\right)$.

The main result of this paper, Theorem 2, provides necessary and sufficient conditions for Problem 1 to be solvable.

\footnotetext{
1 See Section 6 and Definition 7.
} 


\section{Preliminary results}

In this section we investigate the problem of restricting the control-affine system (1) to nested sets $S_{1} \supset S_{2}$ satisfying Assumption 1.

Problem 2. (Restricting control-affine systems to nested sets problem). Given the control system (1), nested sets $S_{1} \supset S_{2}$ satisfying Assumption 1 , and a point $\bar{x} \in S_{2}$, find, if possible, a diffeomorphism $\Xi: U \rightarrow \Xi(U) \subseteq$ $\mathbb{R}^{s_{2}} \times \mathbb{R}^{s_{1}-s_{2}} \times \mathbb{R}^{n-s_{1}}, x \mapsto(\zeta, \mu, \xi)$, and a regular feedback transformation $(\alpha, \beta)$ valid in a neighbourhood $U \subseteq \mathbb{R}^{n}$ of $\bar{x}$, such that (1) is feedback equivalent to

$$
\begin{aligned}
\dot{\zeta} & =f_{1}(\zeta, \mu, \xi)+g_{11}(\zeta, \mu, \xi) v^{\|}+g_{12}(\zeta, \mu, \xi) v^{\|, \pitchfork}+g_{13}(\zeta, \mu, \xi) v^{\pitchfork} \\
\dot{\mu} & =f_{2}(\zeta, \mu, \xi)+g_{21}(\zeta, \mu, \xi) v^{\|}+g_{22}(\zeta, \mu, \xi) v^{\|, \pitchfork}+g_{23}(\zeta, \mu, \xi) v^{\pitchfork} \\
\dot{\xi} & =f_{3}(\zeta, \mu, \xi)+g_{31}(\zeta, \mu, \xi) v^{\|}+g_{32}(\zeta, \mu, \xi) v^{\|, \pitchfork}+g_{33}(\zeta, \mu, \xi) v^{\pitchfork}
\end{aligned}
$$

where

$$
\begin{gathered}
\Xi\left(S_{1} \cap U\right)=\{(\zeta, \mu, \xi) \in \Xi(U): \xi=0\}, \\
\Xi\left(S_{2} \cap U\right)=\{(\zeta, \mu, \xi) \in \Xi(U): \xi=0, \mu=0\},
\end{gathered}
$$

$f_{3}(\zeta, \mu, 0)=0, g_{31}(\zeta, \mu, 0)=0, g_{32}(\zeta, \mu, 0)=0, f_{2}(\zeta, 0,0)=0$, and $g_{21}(\zeta, 0,0)=$ 0 .

The normal form (7) features two types of decomposition similar to those in (3). However, unlike (3), we do not require the transversal dynamics to $S_{2}$, restricted to $S_{1}$ be linear and controllable. The normal form (7) is useful for understanding the inter-play between the control vector fields $g_{1}, \cdots, g_{m}$ of (1) and the nested sets $S_{1} \supset S_{2}$. That is, $g$ is partitioned into three sub-matrices corresponding to $v^{\|}, v^{\|, \pitchfork}$, and $v^{\pitchfork}$. The impositions on $g_{21}$ and $g_{31}$ mean that the columns of the matrix $\Xi_{\star}(g \beta)$ corresponding to $v^{\|}$are tangent to both $\Xi\left(S_{1} \cap U\right)$ and $\Xi\left(S_{2} \cap U\right)$. The requirement on $g_{32}$ implies that the columns corresponding to $v^{\|, \pitchfork}$ are tangent to $\Xi\left(S_{1} \cap U\right)$ but not $\Xi\left(S_{2} \cap U\right)$. Finally, the requirements on $f_{2}, f_{3}$, imply that the vector field $\Xi_{\star}(f+g \alpha)=\left(f_{1}, f_{2}, f_{3}\right)$ is tangent to both $\Xi\left(S_{1} \cap U\right)$ and $\Xi\left(S_{2} \cap U\right)$. As with the normal form (3), substituting $\xi=0$ and $v^{\pitchfork}=0$, the dynamics of (1) restricted to $\Xi\left(S_{1} \cap U\right)$ are

$$
\begin{aligned}
& \dot{\zeta}=f_{1}(\zeta, \mu, 0)+g_{11}(\zeta, \mu, 0) v^{\|}+g_{12}(\zeta, \mu, 0) v^{\|, \pitchfork} \\
& \dot{\mu}=f_{2}(\zeta, \mu, 0)+g_{21}(\zeta, \mu, 0) v^{\|}+g_{22}(\zeta, \mu, 0) v^{\|, \pitchfork} .
\end{aligned}
$$

The tangential dynamics on $S_{2}$ are the same as (6). In principle, the normal form (7) may facilitate the design of control laws to stabilize $\xi=0$ and $\mu=0$. However, a drawback of (7) is that the dynamics remain nonlinear and it may not be clear how to proceed with control design. The aforementioned partition of $g$ is closely related to the properties of the distributions

$$
\begin{aligned}
& P:=G_{0} \cap T S_{2} \\
& Q:=\left[G_{0} \cap T S_{2}\right]^{\perp} \cap\left[G_{0} \cap T S_{1}\right] \\
& R:=\left[G_{0} \cap T S_{1}\right]^{\perp} \cap G_{0}
\end{aligned}
$$


and the integer-valued functions $\nu, \rho: S_{2} \rightarrow \mathbb{Z}, \sigma: S_{1} \rightarrow \mathbb{Z}$

$$
\begin{aligned}
\nu(x) & :=\operatorname{dim}\left(T_{x} S_{2} \cap G_{0}(x)\right) \\
\rho(x) & :=\operatorname{dim}\left(T_{x} S_{1} \cap G_{0}(x)\right)-\nu(x) \\
\sigma(x) & :=\operatorname{dim}\left(T_{x} S_{1}+G_{0}(x)\right)-s_{1} .
\end{aligned}
$$

The values of (11) equal the dimensions of the distributions (10) and the sizes of the sub-matrices corresponding to $v^{\|}, v^{\|, \pitchfork}$, and $v^{\pitchfork}$ in $(7)$.

Proposition 1 For all $p \in S_{1}, q \in S_{2}, \operatorname{dim}(P(q))=\nu(q), \operatorname{dim}(Q(q))=\rho(q)$ and $\operatorname{dim}(R(p))=\sigma(p)$.

Proposition 1, whose proof is given in Appendix A, motivates Definition 4.

Definition 4 A point $\bar{x} \in S_{2}$ is a regular point of the distributions (10) if there exists an open set $V_{1} \subseteq S_{1}$ containing $\bar{x}$ such that for all $p \in V_{1}$, $q \in V_{1} \cap S_{2}$, the functions $\sigma(p), \nu(q), \rho(q)$ are constant.

Remark 2 Under Assumption 1 the topology of $S_{1}$ is its subspace topology as a subset of $\mathbb{R}^{n}$. Thus for each open set $V_{1} \subseteq S_{1}$ there exists an open set $U \subseteq \mathbb{R}^{n}$ such that $V_{1}=U \cap S_{1}$.

The next proposition provides a computationally tractable way of checking the regularity of the distributions (10).

Proposition $2 A$ point $\bar{x} \in S_{2}$ is a regular point of (10) if and only if $\operatorname{dim}\left(T_{x} S_{1} \cap G_{0}(x)\right)$ and $\operatorname{dim}\left(T_{x} S_{2} \cap G_{0}(x)\right)$ are constant in, respectively, open sets $V_{1} \subseteq S_{1}, V_{2} \subseteq S_{2}$ containing $\bar{x}$.

Remark 3 When $\bar{x}$ is a regular point of (10), $T S_{1} \cap G_{0}$ and $T S_{2} \cap G_{0}$ can be viewed as vector bundles over the base spaces $V_{1}$ and $V_{2}$, receptively. In the remainder of this paper we forgo this formality and refer to them as distributions. It is easy to show that if any two of the functions in (11) are constant in an open subset of $S_{2}$, then the remaining function is also constant on this set. Furthermore, if $x$ is a regular point of (11) then $\nu(x)+\rho(x)+\sigma(x)=m$.

Proposition $3 A$ point $\bar{x} \in S_{2}$ is a regular point of (10) if and only if there exists an open set $V_{1} \subseteq S_{1}$ containing $\bar{x}$ such that the distributions (10) are smooth and non-singular in $V_{1}$ and $V_{1} \cap S_{2}$.

Lemma 1 The values of the functions (11) are invariant under coordinate and feedback transformation.

The proofs of Propositions 2 and 3 and Lemma 1 are in Appendix A. The following lemma generalizes slice coordinates for two nested embedded submanifolds. 
Definition 5 ([26]) Let $N$ be a smooth manifold with dimension $n$ and let $(U, \varphi)$ be a smooth chart on $N$. If $S$ is a subset of $N$ such that

$$
\varphi(S \cap U)=\left\{x \in U: \varphi_{k+1}(x)=c_{k+1}, \cdots, \varphi_{n}(x)=c_{n}\right\}
$$

for real constants $c_{k+1}, \cdots, c_{n}$, then $S$ is a $k$-slice of $U$.

Lemma 2 Let $S_{1} \supset S_{2}$ be two smooth embedded submanifolds of $\mathbb{R}^{n}$. For all $\bar{x} \in S_{2}$ there exists an open set $U \subseteq \mathbb{R}^{n}$ such that $S_{1}$ and $S_{2}$ are, respectively, $s_{1}$-slices and $s_{2}$-slices of $U$.

Proof. Let $\bar{x} \in S_{2}$ be arbitrary. Since $S_{1} \subseteq \mathbb{R}^{n}$ is an embedded submanifold there exist slice coordinates $\left(V_{1}, \psi\right)$ for $\mathbb{R}^{n}$ with $\bar{x} \in V_{1}$ such that

$$
\psi\left(S_{1} \cap V_{1}\right)=\left\{x \in V_{1}: \psi_{s_{1}+1}(x)=c_{s_{1}+1}, \cdots, \psi_{n}(x)=c_{n}\right\}
$$

where, without loss of generality, we take the constants $c_{i}$ to be zero. Let $\pi_{1}: \mathbb{R}^{n} \rightarrow \mathbb{R}^{n-s_{1}}$ denote the projection onto the last $n-s_{1}$ factors, i.e, $\pi_{1}(x)=\left(x_{s_{1}+1}, \cdots, x_{n}\right)$. Define $\Phi_{1}: V_{1} \rightarrow \mathbb{R}^{n-s_{1}}, x \mapsto \pi_{1} \circ \psi(x)$. Then $\Phi_{1}$ is a submersion and

$$
\psi\left(S_{1} \cap V_{1}\right)=\left\{x \in V_{1}: \Phi_{1}(x)=0\right\} .
$$

This construction is summarized in the following commutative diagram

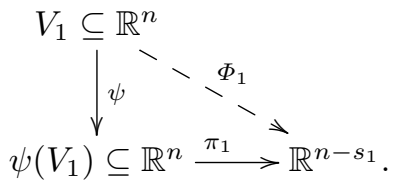

We now apply a similar construction to $S_{2}$. Let $\left(V_{2}, \varphi\right)$ be slice coordinates for $\mathbb{R}^{n}$ with $\bar{x} \in V_{2}$ and let $\pi_{2}: \mathbb{R}^{n} \rightarrow \mathbb{R}^{n-s_{2}}$ be the projection onto the last $n-s_{2}$ factors. Then, letting $\Phi_{2}:=\pi_{2} \circ \phi$ we have

$$
\varphi\left(S_{2} \cap V_{2}\right)=\left\{x \in V_{2}: \Phi_{2}(x)=0\right\} .
$$

and the commutative diagram

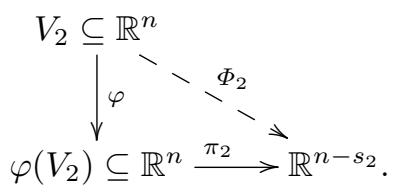

Let $U:=V_{1} \cap V_{2}$ and note that $\bar{x} \in U$. Since $\Phi_{1}$ and $\Phi_{2}$ are submersions we have that, for all $x \in U, \operatorname{rank}\left(\mathrm{d} \Phi_{1}\right)=n-s_{1}$ and $\operatorname{rank}\left(\mathrm{d} \Phi_{2}\right)=n-s_{2}$. Furthermore, by [26, Lemma 8.15], for all $x \in S_{2} \cap U$, Ker $\mathrm{d} \Phi_{1, x}=T_{x} S_{1}$ and $\operatorname{Ker} \mathrm{d} \Phi_{2, x}=T_{x} S_{2}$. Therefore Ker $\mathrm{d} \Phi_{2, x} \subset \operatorname{Ker} \mathrm{d} \Phi_{1, x}$ and

$$
\operatorname{rank}\left[\begin{array}{c}
\mathrm{d} \Phi_{1, x} \\
\mathrm{~d} \Phi_{2, x}
\end{array}\right]=\operatorname{rank}\left[\mathrm{d} \Phi_{2, x}\right]=n-s_{2} .
$$


This allows us to construct a submersion $\Phi: U \rightarrow \mathbb{R}^{n-s_{2}}$. We take the last $n-s_{1}$ components of $\Phi$ to be the function $\Phi_{1}$. From (12) we conclude that in the set $\Phi_{2}=\left\{\varphi_{s_{2}+1}, \cdots, \varphi_{n}\right\}$ it is possible to find $s_{1}-s_{2}$ functions, without of loss of generality $\left\{\varphi_{s_{2}+1}, \cdots, \varphi_{s_{1}}\right\}=$ : $\bar{\Phi}_{2}$, with the property that the $n-s_{2}$ differentials $\mathrm{d} \varphi_{s_{2}+1}, \cdots, \mathrm{d} \varphi_{s_{1}}, \mathrm{~d} \psi_{s_{1}+1}, \cdots, \mathrm{d} \psi_{n}$ are linearly independent at $\bar{x}$. Let $\Phi:=\left(\bar{\Phi}_{2}, \Phi_{1}\right)$.

Since, $\mathrm{d} \Phi(\bar{x})$ has rank $n-s_{2}$ it has some $\left(n-s_{2}\right) \times\left(n-s_{2}\right)$ minor with non-zero determinant. By re-ordering the coordinates we assume that it is the minor corresponding to the first $n-s_{2}$ rows and columns of $\mathrm{d} \Phi(\bar{x})$. Relabel the coordinates as $(y, z)=\left(x_{1}, \cdots, x_{n-s_{2}}, x_{n-s_{2}+1}, \cdots, x_{n}\right)$ in $\mathbb{R}^{n}$. Define $\Xi$ : $U \rightarrow \mathbb{R}^{n}$ by $\Xi(y, z):=(z, \Phi(y, z))$. Its total derivative at $\bar{x}$ is

$$
\mathrm{d} \Xi(\bar{x})=\left[\begin{array}{cc}
0 & I_{s_{2}} \\
\frac{\partial \Phi_{i}}{\partial y_{j}} & \frac{\partial \Phi_{i}}{\partial z_{j}}
\end{array}\right],
$$

which is non-singular because its columns are independent. Therefore, by the inverse function theorem [26, Theorem 7.6], by possibly shrinking $U, \Xi \in$ Diff $(U)$. In the chart $(U, \Xi)$ of $\mathbb{R}^{n}$ we have

$$
\Xi\left(S_{1} \cap U\right)=\left\{x \in U: \Xi_{s_{1}+1}(x)=\cdots=\Xi_{n}(x)=0\right\}
$$

and

$$
\Xi\left(S_{2} \cap U\right)=\left\{x \in U: \Xi_{s_{2}+1}(x)=\cdots=\Xi_{n}(x)=0\right\} .
$$

Definition 6 ([26]) A retraction of a topological space $X$ onto a subspace $A \subset X$ is a continuous map $r: X \rightarrow A$ such that $\left.r\right|_{A}$ is the identity map of $A$.

The tubular neighbourhood theorem [26, Theorem 10.19] states that every embedded submanifold $M$ of $\mathbb{R}^{n}$ has a tubular neighbourhood $\mathcal{N}(M)$. It follows [26, Proposition 10.20] that if $\mathcal{N}(M)$ is a tubular neighbourhood of an embedded submanifold $M \subset \mathbb{R}^{n}$, there exists a smooth retraction of $\mathcal{N}(M)$ onto $M$. In this paper we use a simpler, local version of these ideas.

Lemma 3 Let $M \subset \mathbb{R}^{n}$ be an m-dimensional embedded submanifold of $\mathbb{R}^{n}$. Then, for every $x \in M$ there exist a neighbourhood $U$ of $x$ in $\mathbb{R}^{n}$ and a smooth retraction $r: U \rightarrow M \cap U$.

Proof. Let $\mathcal{N}(M)$ be a tubular neighbourhood of $M$. By [26, Proposition 10.20] there exists a smooth retraction $r: \mathcal{N}(M) \rightarrow M$. Let $U \subseteq \mathcal{N}(M)$ be an open set containing $x$. Then restriction $\left.r\right|_{U}$ is a smooth retraction of $U$ to $M \cap U$.

Lemma 4 Consider two sets $S_{1}$ and $S_{2}$ satisfying Assumption 1 and let $\bar{x} \in$ $S_{2}$ be a regular point of (10). There exists an open set $U \subseteq \mathbb{R}^{n}$ containing $\bar{x}$ and a smooth feedback $\alpha: U \rightarrow \mathbb{R}^{m}$ such that $\left.(f+g \alpha)\right|_{S_{1} \cap U}$ is tangent to $S_{1} \cap U$ and $\left.(f+g \alpha)\right|_{S_{2} \cap U}$ is tangent to $S_{2} \cap U$. 
Proof. Apply Lemma 2 to obtain an open set $U \subseteq \mathbb{R}^{n}$ containing $\bar{x}$ and maps $\Phi_{1}$ and $\bar{\Phi}_{2}$ such that $V_{1}=\Phi_{1}^{-1}(0)$ and $V_{2}=\left(\bar{\Phi}_{2}, \bar{\Phi}_{1}\right)^{-1}(0)$ where $V_{1}:=S_{1} \cap U$ and $V_{2}:=S_{2} \cap U$. Since $S_{1}$ is a controlled-invariant submanifold there exists a smooth state feedback $\alpha_{1}: V_{1} \rightarrow \mathbb{R}^{m}$ such that

$$
\left(\forall x \in V_{1}\right) \quad \mathrm{d} \Phi_{1}(x)\left(f(x)+g(x) \alpha_{1}(x)\right)=0 .
$$

Similarly, since $S_{2}$ is a controlled-invariant submanifold there exists a smooth state feedback $\alpha_{2}: V_{2} \rightarrow \mathbb{R}^{m}$ such that

$$
\left(\forall x \in V_{2}\right) \quad\left[\begin{array}{l}
\mathrm{d} \bar{\Phi}_{2}(x) \\
\mathrm{d} \Phi_{1}(x)
\end{array}\right]\left(f(x)+g(x) \alpha_{2}(x)\right)=0 .
$$

We now modify $\alpha_{1}$ so that the resulting state feedback simultaneously satisfies (13) and (14). We have that

$$
\begin{aligned}
\left(\forall x \in V_{2}\right) \quad & \left.\mathrm{d} \Phi_{1}(x)\left(f(x)+g(x) \alpha_{2}(x)\right)\right|_{V_{2}}-\left.\mathrm{d} \Phi_{1}(x)\left(f(x)+g(x) \alpha_{1}(x)\right)\right|_{V_{2}}=0 \\
& \left.\Rightarrow \mathrm{d} \Phi_{1}(x) g(x)\left(\alpha_{2}(x)-\alpha_{1}(x)\right)\right|_{V_{2}}=0 .
\end{aligned}
$$

Since $\alpha_{1}$ and $\alpha_{2}$ are both smooth, there exists a smooth $\hat{v}(x) \in \operatorname{Ker}\left(\left.\mathrm{d} \Phi_{1}(x) g(x)\right|_{V_{2}}\right)$ such that, for all $x \in V_{2}, \alpha_{2}(x)=\left.\alpha_{1}(x)\right|_{V_{2}}+\hat{v}(x)$. We have that

$$
\begin{aligned}
\left(\forall x \in V_{1}\right) \quad \operatorname{rank}\left(\mathrm{d} \Phi_{1}(x) g(x)\right) & =\operatorname{rank} g(x)-\operatorname{dim}\left(\operatorname{Ker} \mathrm{d} \Phi_{1}(x) \cap \operatorname{rank} g(x)\right) \\
& =\operatorname{dim} G_{0}(x)-\operatorname{dim}\left(T_{x} S_{1} \cap G_{0}(x)\right) .
\end{aligned}
$$

By hypothesis, $\bar{x}$ is a regular point of (10) and by Proposition 2, by possibly shrinking $V_{1}, \operatorname{dim}\left(T_{x} S_{1} \cap G_{0}(x)\right)$ is constant and $\operatorname{dim} G_{0}(x)$ is constant. Thus, $\operatorname{rank}\left(\mathrm{d} \Phi_{1}(x) g(x)\right)$ is constant on $V_{1}$. It implies that $\operatorname{dim}\left(\operatorname{Ker}\left(\mathrm{d} \Phi_{1}(x) g(x)\right)\right)$ is also constant on $V_{1}$. Assume that $\operatorname{dim}\left(\operatorname{Ker}\left(\mathrm{d} \Phi_{1}(x) g(x)\right)\right)=q$. By [16, Lemma 1.3.1], there exists a set $\left\{v_{1}, \cdots, v_{q}\right\}$ of smooth vector fields defined on $V_{1}$ such that at each $x \in V_{1}$, the vectors $v_{1}(x), \cdots, v_{q}(x)$ are linearly independent and

$$
\left(\forall x \in V_{1}\right) \quad \operatorname{Ker}\left(\mathrm{d} \Phi_{1}(x) g(x)\right)=\operatorname{span}\left\{v_{1}(x), \cdots, v_{q}(x)\right\} .
$$

Thus we can write

$$
\hat{v}(x)=\sum_{i=1}^{q} \hat{c}_{i}(x) v_{i}(x) .
$$

where $\hat{c}_{i}: V_{2} \rightarrow \mathbb{R}$ are smooth real-valued functions. Apply Lemma 3 and, by possibly shrinking $U$, introduce a retraction $r_{1}: V_{1} \rightarrow V_{2}$ of $V_{1}$ onto $V_{2}$ and define

$$
\begin{aligned}
c_{i}: V_{1} & \rightarrow \mathbb{R} \\
x & \mapsto \hat{c}_{i} \circ r_{1}(x) .
\end{aligned}
$$

and

$$
v(x)=\sum_{i=1}^{q} c_{i}(x) v_{i}(x)
$$


Let $\alpha^{\prime}:=\alpha_{1}+v$. It solves equation (13) since

$\left(\forall x \in V_{1}\right) \mathrm{d} \Phi_{1}(x)\left(f(x)+g(x) \alpha^{\prime}(x)\right)=\mathrm{d} \Phi_{1}(x)\left(f(x)+g(x) \alpha_{1}(x)\right)+\mathrm{d} \Phi_{1}(x) g(x) v(x)=0$.

Similarly it can be verified that it solves equation (14). Again, applying Lemma 3 we introduce a retraction $r_{2}: U \rightarrow V_{1}$ of $U$ into $V_{1}$ and define

$$
\begin{aligned}
\alpha: U & \rightarrow \mathbb{R}^{m} \\
x & \mapsto \alpha^{\prime} \circ r_{2}(x) .
\end{aligned}
$$

The state feedback $\alpha$ has the desired property.

Remark 4 For LTI control systems [37, Lemma 5.7] asserts that, for nested $(A, B)$-invariant subspaces $\mathscr{S}_{1} \supset \mathscr{S}_{2}$, if $F_{0}$ is a friend of $\mathscr{S}_{2}$ there always exists a mutual friend $F$ such that $\left.F\right|_{\mathscr{S}_{2}}=\left.F_{0}\right|_{\mathscr{S}_{2}}$. Lemma 4 recovers this result because the regularity assumption always holds for LTI systems.

\subsection{Solution to Problem 2}

Theorem 1 Problem 2 is solvable at $\bar{x} \in S_{2}$ if and only if $\bar{x}$ is a regular point of (10).

Proof. Assume that Problem 2 is solvable at $\bar{x} \in S_{2}$. Then there exists a neighbourhood $U \subseteq \mathbb{R}^{n}$ containing $\bar{x}$, a feedback transformation $(\alpha, \beta)$ defined on $U$, and a diffeomorphism $\Xi \in \operatorname{Diff}(U)$ such that (1) is locally feedback equivalent to (7). Let $V_{2}:=S_{2} \cap U, V_{1}:=S_{1} \cap U$, denote by $(\zeta, \mu, 0)=\Xi(x)$ the image of a point $x \in V_{1}$ and by $(\zeta, 0,0)=\Xi(x)$ the image of a point $x \in V_{2}$ under the map $\Xi$, and let $\hat{g}:=\Xi_{\star}(g \beta), \hat{G}_{0}:=\operatorname{span}\left\{\hat{g}_{1}, \cdots, \hat{g}_{m}\right\}$. In $(\zeta, \mu, \xi)$ coordinates the value of $\sigma$ in (11b) at an arbitrary point $(\zeta, \mu, 0) \in \Xi\left(V_{1}\right)$ equals

$$
\sigma(\zeta, \mu, 0)=\operatorname{dim}\left(\operatorname{Im}\left[\begin{array}{cc}
I_{s_{1}} & \star \\
0 & g_{33}(\zeta, \mu, 0)
\end{array}\right]\right)-s_{1}=\operatorname{rank}\left(g_{33}(\zeta, \mu, 0)\right) .
$$

We now argue that $g_{33}$ has full column rank. The equality above implies that the number of columns in $g_{33}(\zeta, \mu, 0)$ is greater than or equal to $\sigma(\zeta, \mu, 0)$. Suppose, by way of contradiction, that $g_{33}$ has $\sigma(\zeta, \mu, 0)+1$ columns. Then, since there are $m$ inputs

$$
\operatorname{rank}\left[\begin{array}{ll}
g_{11}(\zeta, \mu, 0) & g_{12}(\zeta, \mu, 0) \\
g_{21}(\zeta, \mu, 0) & g_{22}(\zeta, \mu, 0)
\end{array}\right] \leq m-\sigma(\zeta, \mu, 0)-1 .
$$

But this means that $\operatorname{dim}\left(\hat{G}_{0}(\zeta, \mu, 0)\right) \leq m-1$ which is a contradiction since $(\alpha, \beta)$ is a regular feedback transformation and $\operatorname{dim}\left(G_{0}(x)\right)=m$. Thus $g_{33}(\zeta, \mu, 0)$ has full column rank. This shows that at an arbitrary point $(\zeta, \mu, 0)$, the integer function $\sigma$ is equal to the number of columns in $g_{33}$. Since $(\zeta, \mu, 0)$ is arbitrary, we conclude that $\sigma$ is constant on $\Xi\left(V_{1}\right)$. 
Having shown that $g_{33}(\zeta, \mu, 0)$ has full column rank on $\Xi\left(V_{1}\right)$ it follows that $\nu(\zeta, 0,0)$ equals

$\operatorname{dim}\left(T_{(\zeta, 0,0)} \Xi\left(V_{2}\right) \cap \hat{G}_{0}(\zeta, 0,0)=\operatorname{dim}\left(\operatorname{Im}\left[\begin{array}{c}I_{s_{2}} \\ 0\end{array}\right] \cap \operatorname{Im}\left[\begin{array}{rr}g_{11}(\zeta, 0,0) & g_{12}(\zeta, 0,0) \\ 0 & g_{22}(\zeta, 0,0)\end{array}\right]\right)\right.$.

Additionally, since

$$
\operatorname{dim}\left(\left[\begin{array}{c}
I_{s_{2}} \\
0
\end{array}\right]+\operatorname{Im}\left[\begin{array}{cr}
g_{11}(\zeta, 0,0) & g_{12}(\zeta, 0,0) \\
0 & g_{22}(\zeta, 0,0)
\end{array}\right]\right)=\operatorname{rank}\left[\begin{array}{cc}
I_{s_{2}} & \star \\
0 & g_{22}(\zeta, 0,0)
\end{array}\right]
$$

we get that

$$
\nu(\zeta, 0,0)=s_{2}+m-\sigma(\zeta, 0,0)-\left(\operatorname{rank} g_{22}(\zeta, 0,0)+s_{2}\right) .
$$

Using the above expression for $\nu$ and the identity, see Remark $3, \nu+\rho+\sigma=m$ at $(\zeta, 0,0)$ we obtain $\rho(\zeta, 0,0)=\operatorname{rank} g_{22}(\zeta, 0,0)$. Using the same reasoning as earlier, one can show $g_{22}(\zeta, 0,0)$ has full column rank. Thus, at $(\zeta, 0,0)$, the integer function $\rho$ is equal to the number of columns in $g_{22}$ which is constant at any point $(\zeta, 0,0) \in \Xi\left(V_{2}\right)$. Finally, if two of the functions in (11) are constant on $\Xi\left(V_{2}\right)$, then so is the third. By Lemma 1 the values of (11) are invariant under feedback and coordinate transformation which shows that $\bar{x}$ is a regular point of (10).

Assume that $\bar{x} \in S_{2}$ is a regular point of (10). By Proposition 3 the distribution $R$ in (10) is smooth and non-singular in a neighbourhood $V_{1} \subseteq S_{1}$ containing $\bar{x}$ and the distributions $P$ and $Q$ in (10) are smooth and nonsingular in $V_{2}=V_{1} \cap S_{2}$. As a result, there exist local generators $\hat{p}_{i}: V_{2} \rightarrow$ $\mathbb{R}^{n}, i \in\{1, \cdots, \nu\}, \hat{q}_{i}: V_{2} \rightarrow \mathbb{R}^{n}, i \in\{1, \cdots, \rho\}$, and $\hat{r}_{i}: V_{1} \rightarrow \mathbb{R}^{n}$, $i \in\{1, \cdots, \sigma\}$ such that, for all $x \in V_{2} P(x)=\operatorname{span}\left\{\hat{p}_{1}, \cdots, \hat{p}_{\nu}\right\}(x)$ and $Q(x)=\operatorname{span}\left\{\hat{q}_{1}, \cdots, \hat{q}_{\rho}\right\}(x)$ and for all $x \in V_{1} R(x)=\operatorname{span}\left\{\hat{r}_{1}, \cdots, \hat{r}_{\sigma}\right\}(x)$. Next, applying Lemma 3 we introduce a retraction $r_{1}: U \rightarrow V_{1}$ of an open set $U \subseteq \mathbb{R}^{n}, \bar{x} \in U$, onto $V_{1}$ and a retraction $r_{2}: U \rightarrow V_{2}$ of an open set $U \subseteq \mathbb{R}^{n}$, $\bar{x} \in U$, onto $V_{2}$ and define

$$
\begin{aligned}
& \begin{array}{l}
p_{i}: U \rightarrow \mathbb{R}^{n} \\
x \mapsto \hat{p}_{i} \circ r_{2}(x) \quad i \in\{1, \cdots, \nu\}
\end{array} \\
& \begin{array}{l}
q_{i}: U \rightarrow \mathbb{R}^{n} \\
x \mapsto \hat{q}_{i} \circ r_{2}(x)
\end{array} \quad i \in\{1, \cdots, \rho\} \\
& r_{i}: U \rightarrow \mathbb{R}^{n} \\
& x \mapsto \hat{r}_{i} \circ r_{1}(x) \quad i \in\{1, \cdots, \sigma\}
\end{aligned}
$$

so that the local generators of $P(x), Q(x)$, and $R(x)$ are now defined on $U$. We set up the following equations

$$
\begin{aligned}
& {\left[p_{1} \cdots p_{\nu}\right]=\left[g_{1} \cdots g_{m}\right] \beta_{1}} \\
& {\left[q_{1} \cdots q_{\rho}\right]=\left[g_{1} \cdots g_{m}\right] \beta_{2}}
\end{aligned}
$$




$$
\left[r_{1} \cdots r_{\sigma}\right]=\left[g_{1} \cdots g_{m}\right] \beta_{3}
$$

where $\beta_{1}: U \rightarrow \mathbb{R}^{m \times \nu}, \beta_{2}: U \rightarrow \mathbb{R}^{m \times \rho}$, and $\beta_{3}: U \rightarrow \mathbb{R}^{m \times \sigma}$ are unknown matrices. Since, $P \subseteq G_{0}$ and both are constant dimensional, by possibly shrinking $U$, there exists a unique smooth solution $\beta_{1}$ to (15a). Similarly, by shrinking $U$, we can find $\beta_{2}$ and $\beta_{3}$ in equations (15b) and (15c), respectively. Define $\left[\beta_{1} \beta_{2} \beta_{3}\right]=: \beta: U \rightarrow \mathrm{GL}(m, \mathbb{R})$. Since $P(x), Q(x)$, and $R(x)$ span independent subspaces for each $x \in U$, the matrix $\beta$ is non-singular.

By Lemma 4 there exists a feedback $\alpha: U \rightarrow \mathbb{R}^{m}$ defined, without loss of generality, on $U$ such that $\left.(f+g \alpha)\right|_{S_{1} \cap U}$ is tangent to $V_{1}:=S_{1} \cap U$ and $\left.(f+g \alpha)\right|_{S_{2} \cap U}$ is tangent to $V_{2}:=S_{2} \cap U$. The pair $(\alpha, \beta)$ is the desired feedback transformation. Applying it to (1) yields

$$
\dot{x}=f(x)+g(x) \alpha(x)+g(x) \beta_{1}(x) v^{\|}+g(x) \beta_{2}(x) v^{\|, \pitchfork}+g(x) \beta_{3}(x) v^{\pitchfork}
$$

where $v^{\|} \in \mathbb{R}^{\nu}, v^{\|, \pitchfork} \in \mathbb{R}^{\rho}$, and $v^{\pitchfork} \in \mathbb{R}^{\sigma}$.

By Lemma 4 the vector field $\left.(f(x)+g(x) \alpha(x))\right|_{V_{2}}$ is tangent to both $V_{1}$ and $V_{2}$. Columns of $\left.g \beta_{1}\right|_{V_{2}}$ are the local generators of $P$ thus are tangent to $V_{2}$. the columns of $\left.g \beta_{2}\right|_{V_{2}}$ are local generators of $Q$, so are tangent to $V_{1}$ and not $V_{2}$. Finally, columns of $\left.g \beta_{3}\right|_{V_{2}}$ are local generators of $R$, so are tangent to neither $V_{1}$ nor $V_{2}$. Select $\Xi$ to be the diffeomorphism from Lemma 2. Applying the coordinate transformation $\Xi$ to (16) yields the desired normal form (7).

The following example is intended to shed light on the concepts discussed in this section.

Example 1. Consider the control system

$\dot{x}=\left[\begin{array}{c}-x_{2} \\ x_{1}-\left(x_{1}^{2}+x_{2}^{2}-1\right)\left(x_{3}^{2}+x_{4}^{2}-1\right) \\ -x_{4} \\ x_{3}-x_{1}^{2}-x_{2}^{2}-x_{3}^{2}-x_{4}^{2}+2\end{array}\right]+\left[\begin{array}{c}-x_{2} \\ x_{1} \\ 0 \\ 0\end{array}\right] u_{1}+\left[\begin{array}{c}-x_{2}^{2} \\ x_{1} x_{2} \\ -x_{4} \\ x_{3}\end{array}\right] u_{2}+\left[\begin{array}{c}0 \\ 0 \\ x_{2} \\ 0\end{array}\right] u_{3}$,

and two nested sets

$$
S_{1}:=\left\{x \in \mathbb{R}^{4}: x_{1}^{2}+x_{2}^{2}-1=0\right\}, S_{2}:=\left\{x \in S_{1}: x_{3}^{2}+x_{4}^{2}-1=0\right\},
$$

and the point $\bar{x}=(0,1,1,0) \in S_{2}$. The objective is to solve Problem 2 at $\bar{x}$. We first check the conditions of Theorem 1 which requires $\bar{x}$ to be a regular point of (10). Since $S_{1}$ and $S_{2}$ are embedded in $\mathbb{R}^{4}$ as the zero level sets of smooth functions, it is easy to show that

$$
T_{x} S_{1}=\operatorname{span}\left\{\left[\begin{array}{c}
-x_{2} \\
x_{1} \\
0 \\
0
\end{array}\right],\left[\begin{array}{l}
0 \\
0 \\
1 \\
0
\end{array}\right],\left[\begin{array}{l}
0 \\
0 \\
0 \\
1
\end{array}\right]\right\}, \quad T_{x} S_{2}=\operatorname{span}\left\{\left[\begin{array}{c}
-x_{2} \\
x_{1} \\
0 \\
0
\end{array}\right],\left[\begin{array}{c}
0 \\
0 \\
-x_{4} \\
x_{3}
\end{array}\right]\right\} .
$$


We compute $\nu(x)$ and $\rho(x)$ as follows

$$
\nu(x)=\operatorname{rank}\left[\begin{array}{cc}
-x_{2} & -x_{2}^{2} \\
x_{1} & x_{1} x_{2} \\
0 & -x_{4} \\
0 & x_{3}
\end{array}\right], \quad \rho(x)=\operatorname{rank}\left[\begin{array}{ccc}
-x_{2} & -2 & 0 \\
x_{1} & x_{1} x_{2} & 0 \\
0 & -x_{4} & x_{2} \\
0 & x_{3} & 0
\end{array}\right]-\nu(x)
$$

Let $U=\left\{x \in \mathbb{R}^{4}: x_{2} \neq 0\right.$ and $\left.x_{3} \neq 0\right\}$ be a neighbourhood of $\bar{x}=(0,1,1,0)$ where, for all $x \in U, \nu(x)=2$ and $\rho(x)=1$. Since the constancy of any two functions in (11) implies the constancy of the third, $\bar{x}$ is a regular point of (10) and by Theorem 1 Problem 2 is solvable there.

First, we find the feedback transformation $(\alpha, \beta)$. The function $\alpha: U \rightarrow \mathbb{R}^{3}$ is a mutual friend of $S_{1} \cap U$ and $S_{2} \cap U$. Following the procedure in the proof of Lemma 4 we obtain $\alpha(x)=0$. In order to find $\beta$ we compute the distributions (10) of Proposition 3

$$
P(x)=\operatorname{span}\left\{\left[\begin{array}{c}
-x_{2} \\
x_{1} \\
0 \\
0
\end{array}\right],\left[\begin{array}{c}
-x_{1}^{2} \\
x_{1} x_{2} \\
-x_{4} \\
x_{3}
\end{array}\right]\right\}, Q(x)=\operatorname{span}\left\{\left[\begin{array}{c}
0 \\
0 \\
x_{3} \\
x_{4}
\end{array}\right]\right\}, R(x)=0 .
$$

Even though, the distributions (10) are only defined on $S_{1} \cap U$ and $S_{2} \cap U$, the calculated distributions are valid on the entire set $U$ eliminating the need for the retractions in the proof of Theorem 1. Solving equation (15) yields

$$
\beta: U \rightarrow \mathrm{GL}(3, \mathbb{R}), x \mapsto\left[\begin{array}{ccc}
1 & 0 & \frac{-x_{2} x_{4}}{x_{3}} \\
0 & 1 & \frac{x_{4}}{x_{3}} \\
0 & 0 & \frac{x_{3}^{2}+x_{4}^{2}}{x_{2} x_{3}}
\end{array}\right]
$$

We follow the proof of Lemma 2 to find the coordinate transformation $\Xi \in$ Diff $(U)$ to be defined by $x \mapsto\left(x_{1}, x_{3}, x_{3}^{2}+x_{4}^{2}-1, x_{1}{ }^{2}+x_{2}{ }^{2}-1\right)$. Applying the feedback transformation $(\alpha, \beta)$ and coordinate transformation $\Xi$ to (17) we obtain

$$
\begin{aligned}
\dot{\zeta}_{1} & =\left(1+\xi-\zeta_{1}^{2}\right)^{\frac{1}{2}}+\left(1+\xi-\zeta_{1}^{2}\right)^{\frac{1}{2}} v_{1}^{\|}-\left(1+\xi-\zeta_{1}^{2}\right) v_{2}^{\|} \\
\dot{\zeta}_{2} & =\left(1+\mu-\zeta_{2}^{2}\right)^{\frac{1}{2}}+\left(1+\mu-\zeta_{2}^{2}\right)^{\frac{1}{2}} v_{2}^{\|}+\zeta_{2} v^{\|, \pitchfork} \\
\dot{\mu} & =2(\xi+\mu)\left(1+\mu-\zeta_{2}^{2}\right)^{\frac{1}{2}}+2(\mu+1) v^{\|, \pitchfork} \\
\dot{\xi} & =2 \xi \mu\left(1+\xi-\zeta_{1}^{2}\right)^{\frac{1}{2}} .
\end{aligned}
$$

Note that when $\xi=0$, the term $f_{3}(\zeta, \mu, \xi)=2 \xi \mu\left(1+\xi-\zeta_{1}^{2}\right)^{\frac{1}{2}}$ vanishes and $g_{31}$ and $g_{32}$ are identically zero. Also, when $\xi=0$ and $\mu=0$ the terms $f_{2}(\zeta, \mu, \xi)=$ $2(\xi+\mu)\left(1+\mu-\zeta_{2}^{2}\right)^{\frac{1}{2}}$ vanishes and $g_{21}(\zeta, \mu, \xi)$ is identically zero. Thus, the requirements on normal form (7) are satisfied. The dynamics restricted to $S_{1} \cap U$ are obtained by substituting $\xi=0$ in (18) and the dynamics restricted to $S_{2} \cap U$ is obtained by substituting $\xi=0$ and $\mu=0$ in (18). 
4 Solution to partial local nested transverse feedback linearization problem

We are now ready to present the main result of this paper, necessary and sufficient conditions for Problem 1 to be solvable. It is evident that (3) is a refinement of (7) and thus the solvability of Problem 2 is a necessary condition for Problem 1 to be solvable. Thus throughout this section we make the following assumption.

Assumption 2. The point $\bar{x} \in S_{2}$ is a regular point of (10).

Assumption 2 implies, by Theorem 1, that Problem 2 is solvable at $\bar{x}$. Therefore, there exists a regular feedback transformation $(\alpha, \beta)$ such that, control system (1) on a neighbourhood $U \subset \mathbb{R}^{n}$ of $\bar{x}$ writes as (16), re-written here for convenience,

$$
\dot{x}=f(x)+g(x) \alpha(x)+g(x) \beta_{1}(x) v^{\|}+g(x) \beta_{2}(x) v^{\|, \pitchfork}+g(x) \beta_{3}(x) v^{\pitchfork}
$$

where $\left(v^{\|}, v^{\|, \pitchfork}, v^{\pitchfork}\right) \in \mathbb{R}^{\nu} \times \mathbb{R}^{\rho} \times \mathbb{R}^{\sigma}$. Recall that $v^{\pitchfork}=0$ renders $S_{1} \cap U$ invariant; and the vector field $f+g \alpha$ and columns of $g \beta_{1}$ and columns of $g \beta_{2}$ are tangent to $S_{1} \cap U$. Thus, the restriction of (1) with $v^{\pitchfork}=0$ to $S_{1} \cap U$ is well-defined. We introduce the following short hand notation for the restriction

$$
f_{S_{1}}:=\left.(f+g \alpha)\right|_{S_{1} \cap U}, \quad g_{S_{1}}:=\left.\left[g \beta_{1} g \beta_{2}\right]\right|_{S_{1} \cap U} \quad v_{S_{1}}^{\|}:=\left(v^{\|}, v^{\|, \pitchfork}\right) .
$$

Then, the dynamics restricted to $S_{1} \cap U$ are

$$
\dot{x}=f_{S_{1}}(x)+g_{S_{1}}(x) v_{S_{1}}^{\|}
$$

Similar to (2), we associate to (19) a family of distribution $G_{i}^{\|}: S_{1} \cap U \rightarrow$ $T\left(S_{1} \cap U\right) \subseteq T \mathbb{R}^{n}$

$$
G_{i}^{\|}(x):=\operatorname{span}\left\{a d_{f_{S_{1}}}^{j} g_{S_{1}, k}(x): 0 \leq j \leq i, 1 \leq k \leq \nu+\rho\right\} .
$$

Theorem 2 (Main Result) Consider control system (1) and nested sets $S_{1} \supset S_{2}$ satisfying Assumption 1. Let $\bar{x} \in S_{2}$ and suppose that $\operatorname{inv}\left(G_{i}^{\|}\right), i \in$ $\mathbf{s}_{\mathbf{1}}-\mathbf{s}_{\mathbf{2}}-\mathbf{1}$ are regular at $\bar{x} \in S_{2}$. Then, Problem 1 is solvable if and only if

(a) $\bar{x}$ is a regular point of $(10)$

(b) $\operatorname{dim}\left(T_{\bar{x}} S_{2}+G_{s_{1}-s_{2}-1}^{\|}(\bar{x})\right)=s_{1}$

(c) There exists an open set $U \subseteq \mathbb{R}^{n}$ containing $\bar{x}$ such that, for all $i \in$ $\mathbf{s}_{\mathbf{1}}-\mathbf{s}_{\mathbf{2}}-\mathbf{1}$, for all $x \in S_{2} \cap U$

$$
\operatorname{dim}\left(T_{x} S_{2}+G_{i}^{\|}(x)\right)=\left(T_{x} S_{2}+\operatorname{inv}\left(G_{i}^{\|}(x)\right)\right)=\text { constant }
$$


Proof. Suppose that Problem 1 is solvable at $\bar{x} \in S_{2}$. Then Problem 2 is solvable since the normal form (3) is a refinement of the normal form (7). Thus, $\bar{x}$ is a regular point of (10) and condition (a) holds. As a result, the assumption requiring $\operatorname{inv}\left(G_{i}^{\|}\right), i \in \mathbf{s}_{\mathbf{1}}-\mathbf{s}_{\mathbf{2}}-\mathbf{1}$ be regular at $\bar{x}$ is a valid assumption. Moreover, since Problem 1 is solvable at $\bar{x} \in S_{2}$ there exists a neighbourhood $U \subseteq \mathbb{R}^{n}$ of $\bar{x}$, a coordinate transformation $\Xi \in \operatorname{Diff}(U)$, and a feedback transformation $(\alpha, \beta)$ such that (1) is feedback equivalent to (3) in $U$. Define $V_{1}:=\Xi\left(S_{1} \cap U\right)$ and $V_{2}:=\Xi\left(S_{2} \cap U\right)$. The system dynamics restricted to $V_{1}$ are given in (5), and any point in $V_{1}$ and $V_{2}$ is represented by $(\zeta, \mu)$ and $(\zeta, 0)$, respectively. In transformed coordinates we have

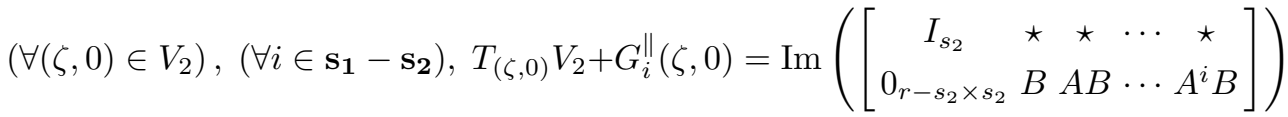

which shows that the dimension of $T_{(\zeta, 0)} V_{2}+G_{i}^{\|}(\zeta, 0)$ is $s_{2}+\operatorname{rank}\left(\left[B \cdots A^{i} B\right]\right)$. Since the pair $(A, B)$ is controllable, $\operatorname{rank}\left(\left[B \cdots A^{s_{1}-s_{2}-1} B\right]\right)=s_{1}-s_{2}$; thus $\operatorname{dim}\left(T_{(\zeta, 0)} V_{2}+G_{s_{1}-s_{2}-1}^{\|}(\zeta, 0)\right)=s_{1}$. Since condition $(a)$ is invariant under coordinate and feedback transformations it follows that condition $(a)$ holds in original coordinates as well.

In $V_{1}$, consider the collection of constant distributions $\Delta_{i}^{\|}, i \in \mathbf{s}_{\mathbf{1}}-\mathbf{s}_{\mathbf{2}}$ given by

$$
\Delta_{i}^{\|}:=\operatorname{Im}\left(I_{s_{2}} \oplus\left[B \cdots A^{i} B\right]\right) .
$$

At each $(\zeta, 0) \in V_{2}, \Delta_{i}^{\|}(\zeta, 0)=T_{(\zeta, 0)} V_{2}+G_{i}^{\|}(\zeta, 0)$; thus, $G_{i}^{\|}(\zeta, 0) \subseteq \Delta_{i}^{\|}$. Furthermore, since each $\Delta_{i}^{\|}$is (trivially) involutive, it follows that $\operatorname{inv}\left(G_{i}^{\|}(\zeta, 0)\right) \subseteq$ $\Delta_{i}^{\|}$. This shows that for all $i \in \mathbf{s}_{\mathbf{1}}-\mathbf{s}_{\mathbf{2}}$

$$
T V_{2}+\operatorname{inv}\left(G_{i}^{\|}(\zeta, 0)\right) \subseteq \Delta_{i}^{\|}(\zeta, 0)=T V_{2}+G_{i}^{\|}(\zeta, 0)
$$

On the other hand $T V_{2}+G_{i}^{\|}(\zeta, 0) \subseteq T V_{2}+\operatorname{inv}\left(G_{i}^{\|}(\zeta, 0)\right)$ always holds which shows that $\Delta_{i}^{\|}(\zeta, 0)=T V_{2}+G_{i}^{\|}(\zeta, 0)=T V_{2}+\operatorname{inv}\left(G_{i}^{\|}(\zeta, 0)\right)$. Condition $(b)$ is invariant under coordinate and feedback transformations; thus it holds in original coordinates.

Conversely, assume that conditions (a), (b), and (c) hold. By condition (a) Problem 2 is solvable and there exists a neighbourhood $U \subseteq \mathbb{R}^{n}$ of $\bar{x}$, a coordinate transformation $\Xi_{1}: U \rightarrow \Xi_{1}(U) \subseteq \mathbb{R}^{s_{2}} \times \mathbb{R}^{s_{1}-s_{2}} \times \mathbb{R}^{n-s_{1}}, x \mapsto(\bar{\zeta}, \bar{\mu}, \bar{\xi})$, and feedback transformation $\left(\alpha_{1}, \beta_{1}\right)$ such that (1) is feedback equivalent to (7) on $U$. Let $\pi_{1}: \mathbb{R}^{n} \rightarrow \mathbb{R}^{s_{1}}$ be the projection to the first $s_{1}$ factors. Let $\bar{V}_{1}:=\pi_{1} \circ \Xi_{1}\left(S_{1} \cap U\right) \subseteq \mathbb{R}^{s_{1}}$ and $\psi=\left.\pi_{1} \circ \Xi_{1}\right|_{S_{1} \cap U}: S_{1} \cap U \rightarrow \bar{V}_{1}$. By [26, Theorem 8.2] $S_{1}$ is a smooth manifold of dimension $s_{1}$ and $\left(S_{1} \cap U, \psi\right)$ is a coordinate chart. Define $\bar{V}_{2}:=\pi_{1} \circ \Xi_{1}\left(S_{2} \cap U\right)$. Since $\bar{V}_{2}=\left\{(\bar{\zeta}, \bar{\mu}) \in \bar{V}_{1}: \bar{\mu}=0\right\}$ we conclude that $\bar{V}_{2}$ is an embedded submanifold of $\bar{V}_{1}$. In this coordinate chart (19) writes as

$$
\begin{aligned}
& \dot{\bar{\zeta}}=\bar{f}_{1}(\bar{\zeta}, \bar{\mu}, 0)+\bar{g}_{11}(\bar{\zeta}, \bar{\mu}, 0) \bar{v}^{\|}+\bar{g}_{12}(\bar{\zeta}, \bar{\mu}, 0) \bar{v}_{2}^{\pitchfork} \\
& \dot{\bar{\mu}}=\bar{f}_{2}(\bar{\zeta}, \bar{\mu}, 0)+\bar{g}_{21}(\bar{\zeta}, \bar{\mu}, 0) \bar{v}^{\|}+\bar{g}_{22}(\bar{\zeta}, \bar{\mu}, 0) \bar{v}_{2}^{\pitchfork} .
\end{aligned}
$$


Since condition (a) holds one can assume $\operatorname{inv}\left(G_{i}^{\|}\right), i \in \mathbf{s}_{\mathbf{1}}-\mathbf{s}_{\mathbf{2}}-\mathbf{1}$ are regular at $\bar{x}$ and considering conditions (b) and (c) all the assumptions and conditions of [30, Theorem 3.2] for $(21)$ with respect to $\bar{V}_{2}$ at $(\bar{\zeta}, 0):=\pi_{1} \circ \Xi_{1}(\bar{x})$ hold. Therefore, by possibly shrinking $\bar{V}_{1}$ (and hence $U$ ), there exist a coordinate transformation $\Xi_{2}: \bar{V}_{1} \rightarrow \Xi_{2}\left(\bar{V}_{1}\right) \subseteq \mathbb{R}^{s_{2}} \times \mathbb{R}^{s_{1}-s_{2}}$, and a regular feedback transformation $\left(\bar{\alpha}_{2}, \bar{\beta}_{2}\right)$, with $\bar{\alpha}_{2}: \overline{\bar{V}}_{1} \rightarrow \mathbb{R}^{\nu+\rho}$ and $\bar{\beta}_{2}: \bar{V}_{1} \rightarrow \mathrm{GL}(\nu+\rho, \mathbb{R})$, such that $(21)$ is feedback equivalent to

$$
\begin{aligned}
& \dot{\zeta}=f_{1}(\zeta, \mu)+g_{11}(\zeta, \mu) v^{\|}+g_{12}(\zeta, \mu) v^{\|, \pitchfork} \\
& \dot{\mu}=A \mu+B v_{2}^{\pitchfork} .
\end{aligned}
$$

Let $\pi_{2}: \mathbb{R}^{n} \rightarrow \mathbb{R}^{n-s_{1}}$ be the projection to the last $n-s_{1}$ factors. We construct a function $\Xi: U \subseteq \mathbb{R}^{n} \rightarrow \Xi(U) \subseteq \mathbb{R}^{s_{2}} \times \mathbb{R}^{s_{1}-s_{2}} \times \mathbb{R}^{n-s_{1}}$ as follows

$$
\Xi:=\left(\Xi_{2} \circ \pi_{1} \circ \Xi_{1}\right) \times\left(\pi_{2} \circ \Xi_{1}\right)=\left[\begin{array}{c}
\Xi_{2} \circ \pi_{1} \\
\pi_{2}
\end{array}\right] \circ \Xi_{1} .
$$

The following diagram illustrates our construction.

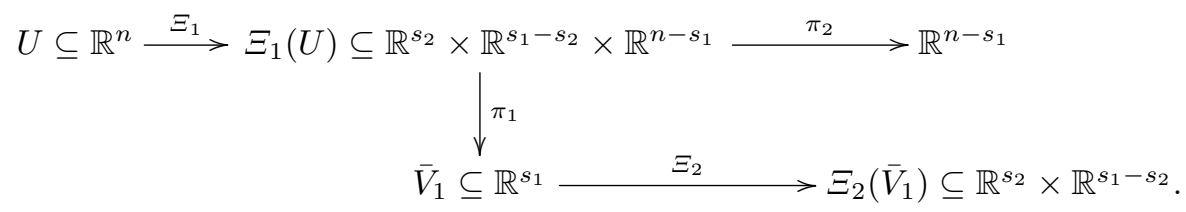

The function $\Xi$ is a well-defined diffeomorphism since at $\bar{x}$

$$
\operatorname{det}(\mathrm{d} \Xi)=\operatorname{det}\left(\left[\begin{array}{cc}
\mathrm{d} \Xi_{2} & 0_{s_{1} \times n-s_{1}} \\
0_{n-s_{1} \times s_{1}} & I_{n-s_{1}}
\end{array}\right]\right) \operatorname{det}\left(\mathrm{d} \Xi_{1}\right) \neq 0 .
$$

Therefore, by the inverse function theorem [26, Theorem 7.6], it is a valid coordinate transformation in a neighbourhood of $\bar{x}$, without loss of generality $U$. In order to construct the feedback transformation we define

$$
\alpha_{2}:=\left[\begin{array}{c}
\bar{\alpha}_{2} \circ \pi_{1} \circ \Xi_{1} \\
0_{\sigma}
\end{array}\right], \quad \beta_{2}:=\left[\begin{array}{cc}
\bar{\beta}_{2} \circ \pi_{1} \circ \Xi_{1} & 0_{(\nu+\rho) \times \sigma} \\
0_{\sigma \times(\nu+\rho)} & I_{\sigma}
\end{array}\right],
$$

where $\alpha_{2}: U \subseteq \mathbb{R}^{n} \rightarrow \mathbb{R}^{m}$ and $\beta: U \subseteq \rightarrow \mathrm{GL}(m, \mathbb{R})$. The feedback transformation $(\alpha, \beta):=\left(\alpha_{1}+\beta_{1} \alpha_{2}, \beta_{1} \beta_{2}\right)$ and $\Xi \in \operatorname{Diff}(U)$ solve Problem 1 .

Remark 5 If the conditions of Theorem 2 hold, then following [30, Theorem 3.1] one can find $\rho$ smooth $\mathbb{R}$-valued functions $\lambda_{1}(\zeta, \mu), \cdots, \lambda_{\rho}(\zeta, \mu)$ defined on $\bar{V}_{1}$, where $\rho$ is given in (11a), such that $(\mathrm{a}) \bar{V}_{2} \subset\left\{(\zeta, \mu) \in \bar{V}_{1}: \lambda_{i}(\zeta, \mu)=0, i \in 1, \cdots, \rho\right\}$

(b) the system $(21)$ with output $y:=\left(\lambda_{1}(\zeta, \mu), \cdots, \lambda_{\rho}(\zeta, \mu)\right)$ has vector relative degree $\left\{k_{1} \cdots, k_{\rho}\right\}$ with $k_{1}+\cdots+k_{\rho}=s_{1}-s_{2}$ at $(\bar{\zeta}, 0)$. Thus, the nested local transverse feedback linearization problem is equivalent to a zero dynamics assignment with well-defined relative degree problem. A semi-constructive procedure to find such functions is presented in the proof of [30, Theorem 3.1]. 


\section{Extension of the main result}

We now outline an extension of the solution to Problem 1. In the extension we seek that the dynamics transversal to the larger set $S_{1}$ also be feedback linearizable. The resulting normal form facilitates the design of controllers to locally stabilize $S_{1}$.

Problem 3. (Local nested transversal feedback linearization problem). Find, if possible, a solution to Problem 1 in which the normal form (3) is replaced by

$$
\begin{aligned}
& \dot{\zeta}=f_{1}(\zeta, \mu, \xi)+g_{11}(\zeta, \mu, \xi) v^{\|}+g_{12}(\zeta, \mu, \xi) v^{\|, \pitchfork}+g_{13}(\zeta, \mu, \xi) v^{\pitchfork} \\
& \dot{\mu}=A \mu+B v^{\|, \pitchfork}+f_{2}(\zeta, \mu, \xi)+g_{21}(\zeta, \mu, \xi) v^{\|}+g_{22}(\zeta, \mu, \xi) v^{\|, \pitchfork}+g_{23}(\zeta, \mu, \xi) v^{\pitchfork} \\
& \dot{\xi}=E \xi+F v^{\pitchfork}
\end{aligned}
$$

where the pair $(E, F)$ is controllable and $F$ is full rank.

In Problem 3 the normal form (3) has been refined because the $\xi$-subsystem is now linear, controllable and decoupled from the $\zeta$ - and $\mu$-subsystems.

Theorem 3 Consider control system (1) and nested sets $S_{1} \supset S_{2}$ satisfying Assumption 1. Let $\bar{x} \in S_{2}$ and assume that the distributions $\operatorname{inv}\left(G_{i}^{\|}\right), \operatorname{inv}\left(G_{j}\right)$, $i \in \mathbf{n}-\mathbf{s}_{\mathbf{1}}-\mathbf{1}, j \in \mathbf{s}_{\mathbf{1}}-\mathbf{s}_{\mathbf{2}}-\mathbf{1}$ are regular at $\bar{x} \in S_{2}$. Then, Problem 3 is solvable if and only if

(a) Problem 1 is solvable.

(b) $\operatorname{dim}\left(T_{\bar{x}} S_{1}+G_{n-s_{1}-1}(\bar{x})\right)=n$.

(c) There exist a neighbourhood $U$ of $\bar{x}$ in $\mathbb{R}^{n}$ such for all $i \in \mathbf{n}-\mathbf{s}_{\mathbf{1}}-\mathbf{1}$, for all $\left(x \in S_{1} \cap U\right)$,

$$
\operatorname{dim}\left(T_{x} S_{1}+G_{i}(x)\right)=\left(T_{x} S_{1}+\operatorname{inv}\left(G_{i}\right)(x)\right)=\text { constant } .
$$

Proof. Assume that Problem 3 is solvable at $\bar{x} \in S_{2}$. The normal form (23) is a refinement of the normal forms (3) and (7). Thus, if Problem 3 is solvable Problems 1 and 2 are solvable. Thus, condition (a) holds. Besides, if Problem 2 is solvable $\bar{x}$ must be a regular point of (10) which implies that it is valid to assume $\operatorname{inv}\left(G_{i}^{\|}\right), i \in \mathbf{n}-\mathbf{s}_{\mathbf{1}}-\mathbf{1}$ are regular at $\bar{x}$. The proof of the necessity of conditions (b) and (c) is easily checked in transformed coordinates using arguments analogous to those in the proof of Theorem 2.

Conversely, assume that conditions (a), (b) and (c) hold. By [30, Theorem 3.2 , since conditions (b) and (c) hold, there exist a neighbourhood $U$ of the point $\bar{x}$, a diffeomorphism $\Xi_{1}: U \rightarrow \Xi_{1}(U) \subset \mathbb{R}^{s_{1}} \times \mathbb{R}^{n-s_{1}}$ and a regular feedback transformation $\left(\alpha_{1}, \beta_{1}\right)$ such that system $(1)$, on $U$, is feedback equivalent to

$$
\begin{aligned}
& \dot{\eta}=f_{0}(\eta, \xi)+g^{\|}(\eta, \xi) v_{1}+g^{\pitchfork}(\eta, \xi) v_{2} \\
& \dot{\xi}=E \xi+F v_{2}
\end{aligned}
$$


where the pair $(E, F)$ is controllable and $\Xi_{1}\left(S_{1} \cap U\right)=\left\{(\eta, \xi) \in \Xi_{1}(U): \xi=0\right\}$. Since Problem 1 is solvable Assumption 2 must hold. Thus, $v_{1} \in \mathbb{R}^{\nu+\rho}$, $v_{2} \in \mathbb{R}^{\sigma}$.

Let $\pi_{1}: \mathbb{R}^{n} \rightarrow \mathbb{R}^{s_{1}}$ be the projection to the first $s_{1}$ factors. Let $\bar{V}_{1}:=$ $\pi_{1} \circ \Xi_{1}\left(S_{1} \cap U\right)$ and $\psi=\left.\pi_{1} \circ \Xi_{1}\right|_{S_{1} \cap U}: S_{1} \cap U \rightarrow \bar{V}_{1}$. By [26, Theorem 8.2] $S_{1}$ is a smooth manifold of dimension $s_{1}$ and $\left(S_{1} \cap U, \psi\right)$ is a coordinate chart. Define $\bar{V}_{2}:=\pi_{1} \circ \Xi_{1}\left(S_{2} \cap U\right)$. Since $\bar{V}_{2}$ is diffeomorphic to $S_{2} \cap U$ it is an embedded submanifold of $\bar{V}_{1}$. In this coordinate chart the restricted dynamics in (19) writes as

$$
\dot{\eta}=f_{0}(\eta, 0)+g^{\|}(\eta, 0) v_{1} .
$$

By condition (a) Problem 1 is solvable and the conditions of Theorem 2 hold. Therefore the assumption that $\operatorname{inv}\left(G_{i}^{\|}\right), i \in \boldsymbol{n}-\boldsymbol{s}_{\mathbf{1}}-\mathbf{1}$ are regular at $\bar{x}$ is well-posed. Thus, all the assumptions and conditions of [30, Theorem 3.2] for (25) with respect to $\bar{V}_{2}$ at $\bar{\eta}:=\pi_{1} \circ \Xi_{1}(\bar{x})$ hold. Therefore, by possibly shrinking $\bar{V}_{1}$ (and hence $U$ ), there exists a coordinate transformation $\Xi_{2}$ : $\bar{V}_{1} \rightarrow \Xi_{2}\left(\bar{V}_{1}\right) \subseteq \mathbb{R}^{s_{2}} \times \mathbb{R}^{s_{1}-s_{2}}$, and a regular feedback transformation $\left(\bar{\alpha}_{2}, \bar{\beta}_{2}\right)$, with $\bar{\alpha}_{2}: \bar{V}_{1} \rightarrow \mathbb{R}^{\nu+\rho}$ and $\bar{\beta}_{2}: \bar{V}_{1} \rightarrow \mathrm{GL}(\nu+\rho, \mathbb{R})$, such that $(25)$ is feedback equivalent to

$$
\begin{aligned}
& \dot{\zeta}=f_{1}(\zeta, \mu)+g_{11}(\zeta, \mu) v^{\|}+g_{12}(\zeta, \mu) v^{\|, \pitchfork} \\
& \dot{\mu}=A \mu+B v^{\|, \pitchfork} .
\end{aligned}
$$

The desired diffeomorphism $\Xi$ is constructed from $\Xi_{1}$ and $\Xi_{2}$ in the same manner as in the proof of Theorem 2. The feedback transformation $(\alpha, \beta)$ is also constructed from $\left(\alpha_{1}, \beta_{1}\right)$ and $\left(\bar{\alpha}_{2}, \bar{\beta}_{2}\right)$ in the same way as in the proof of Theorem 2.

The following example concerning the system from Example 1 illustrates a case in which Problem 1 is solvable while Problem 3 is not.

Example 2. Recall the system, nested sets $S_{1} \supset S_{2}$, and point $\bar{x}=(0,1,1,0)$ from Example 1. Since Problem 2 is solvable at $\bar{x}$ in $U=\left\{x \in \mathbb{R}^{4}: x_{2} \neq 0\right.$ and $\left.x_{3} \neq 0\right\}$ we can easily compute the dynamics restricted to $S_{1} \cap U$ in (19) as

$$
\dot{x}=\left[\begin{array}{c}
-x_{2} \\
x_{1} \\
-x_{4} \\
x_{3}-x_{3}^{2}-x_{4}^{2}+1
\end{array}\right]+\left[\begin{array}{c}
-x_{2} \\
x_{1} \\
0 \\
0
\end{array}\right] v_{1}^{\|}+\left[\begin{array}{c}
-x_{2}^{2} \\
x_{1} x_{2} \\
-x_{4} \\
x_{3}
\end{array}\right] v_{2}^{\|}+\left[\begin{array}{c}
0 \\
0 \\
x_{3} \\
x_{4}
\end{array}\right] v^{\|, \pitchfork} .
$$

Condition (a) of Theorem 2 is satisfied since

$$
\operatorname{dim}\left(T_{\bar{x}} S_{2}+G_{0}^{\|}(\bar{x})\right)=\operatorname{rank}\left[\begin{array}{rrrrr}
-1 & 0 & -1 & -1 & 0 \\
0 & 0 & 0 & 0 & 0 \\
0 & 0 & 0 & 0 & 1 \\
0 & 1 & 0 & 1 & 0
\end{array}\right]=3
$$


Moreover, since

$$
G_{0}^{\|}=\operatorname{span}\left\{\left[\begin{array}{c}
-x_{2} \\
x_{1} \\
0 \\
0
\end{array}\right],\left[\begin{array}{c}
-x_{2}^{2} \\
x_{1} x_{2} \\
-x_{4} \\
x_{3}
\end{array}\right],\left[\begin{array}{c}
0 \\
0 \\
x_{3} \\
x_{4}
\end{array}\right]\right\}
$$

is involutive, condition (b) of Theorem 2 holds. Therefore Problem 1 is solvable at $\bar{x}$. However, since

$$
\operatorname{dim}\left(T_{\bar{x}} S_{1}+G_{0}(\bar{x})\right)=\operatorname{rank}\left[\begin{array}{rrrrrr}
-1 & 0 & 0 & -1 & -1 & 0 \\
0 & 0 & 0 & 0 & 0 & 0 \\
0 & 1 & 0 & 0 & 0 & 1 \\
0 & 0 & 1 & 0 & 1 & 0
\end{array}\right] \neq 4
$$

condition $(a)$ of Theorem 3 is not satisfied and Problem 3 is cannot be solved at $\bar{x}$. We proceed to find the normal form (3) of Problem 1.

In order to find the desired feedback transformation and coordinate transformation we follow the construction in the proof of Theorem 2. The feedback transformation $\left(\alpha_{1}, \beta_{1}\right)$ and the coordinate transformation $\Xi_{1}$ were already found in Example 1. Letting $\xi=0$ in (18), the dynamics (21) are

$$
\begin{aligned}
\dot{\bar{\zeta}}_{1} & =\left(1-\bar{\zeta}_{1}^{2}\right)^{\frac{1}{2}}+\left(1-\bar{\zeta}_{1}^{2}\right)^{\frac{1}{2}} v_{1}^{\|}-\left(1-\bar{\zeta}_{1}^{2}\right) v_{2}^{\|} \\
\dot{\bar{\zeta}}_{2} & =\left(1+\bar{\mu}-\bar{\zeta}_{2}^{2}\right)^{\frac{1}{2}}+\left(1+\bar{\mu}-\bar{\zeta}_{2}^{2}\right)^{\frac{1}{2}} v_{2}^{\|}+\bar{\zeta}_{2} v^{\|, \pitchfork} \\
\dot{\bar{\mu}} & =2 \bar{\mu}\left(1+\bar{\mu}-\bar{\zeta}_{2}^{2}\right)^{\frac{1}{2}}+2(\bar{\mu}+1) v^{\|, \pitchfork} .
\end{aligned}
$$

We employ the results of [30, Theorem 3.2] to find

$$
\Xi_{2}=\mathbb{1}_{3}, \quad\left(\bar{\alpha}_{2}, \bar{\beta}_{2}\right)=\left(\left[\begin{array}{c}
0 \\
0 \\
-\frac{-2 \bar{\mu}\left(1+\bar{\mu}-\bar{\zeta}_{2}^{2}\right)^{\frac{1}{2}}}{2(\bar{\mu}+1)}
\end{array}\right],\left[\begin{array}{lll}
1 & 0 & 0 \\
0 & 1 & 0 \\
0 & 0 & \frac{1}{2(\bar{\mu}+1)}
\end{array}\right]\right)
$$

where $\mathbb{1}_{3}$ is the identity map. It should be noted that $\bar{\mu}+1=x_{3}^{2}+x_{4}^{4}$. Since, $x_{3} \neq 0$ on $U$ it follows that $\bar{\mu}+1 \neq 0$ on $\Xi_{1}(U)$; thus it is not required to shrink the neighbourhood $U$. Next, we find $\pi_{1}=\mathbb{R}^{4} \rightarrow \mathbb{R}^{3},\left(\bar{\zeta}_{1}, \bar{\zeta}_{2}, \bar{\mu}, \bar{\xi}\right) \mapsto\left(\bar{\zeta}_{1}, \bar{\zeta}_{2}, \bar{\mu}\right)$, $\pi_{2}=\mathbb{R}^{4} \rightarrow \mathbb{R}, \quad\left(\bar{\zeta}_{1}, \bar{\zeta}_{2}, \bar{\mu}, \bar{\xi}\right) \mapsto \bar{\xi}$, introduced in the proof of Theorem 2 . Therefore,

$$
\Xi=\left(\Xi_{2} \circ \pi_{1} \circ \Xi_{1}\right) \times\left(\pi_{2} \circ \Xi_{1}\right)=\left(x_{1}, x_{3}, x_{3}^{2}+x_{4}^{2}-1, x_{1}^{2}+x_{2}^{2}-1\right)
$$

and

$$
\alpha_{2}=\left[\begin{array}{c}
0 \\
0 \\
\frac{\left(x_{3}^{2}+x_{4}^{2}-1\right) x_{4}}{x_{3}^{2}+x_{4}^{2}}
\end{array}\right], \quad \beta_{2}=\left[\begin{array}{llc}
1 & 0 & 0 \\
0 & 1 & 0 \\
0 & 0 & \frac{1}{2\left(x_{3}^{2}+x_{4}^{2}\right)}
\end{array}\right]
$$


And finally the feedback transformation $(\alpha, \beta)$ is

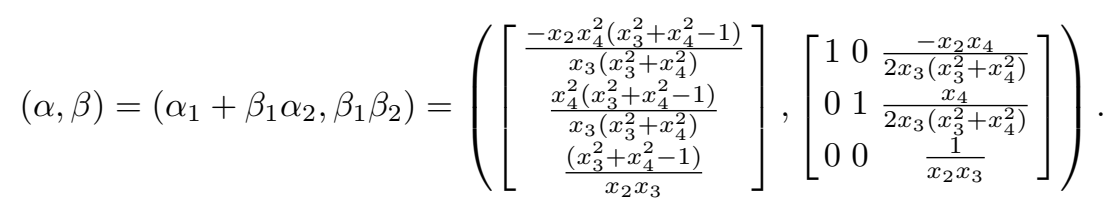

Applying the feedback transformation $(\alpha, \beta)$ and the coordinate transformation $\Xi$ the control system is feedback equivalent to

$$
\begin{aligned}
& \dot{\zeta}_{1}=\left(1+\xi-\zeta_{1}^{2}\right)^{\frac{1}{2}}+\left(1+\xi-\zeta_{1}^{2}\right) v_{1}^{\|}-\left(1+\xi-\zeta_{1}^{2}\right) v_{2}^{\|} \\
& \dot{\zeta}_{2}=\left(1+\mu-\zeta_{2}^{2}\right)^{\frac{1}{2}}+1+\mu-\zeta_{2}^{2 \frac{1}{2}} v_{2}^{\|}+\frac{\zeta_{2}}{2(\mu+1)} v^{\|, \pitchfork} \\
& \dot{\mu}=2 \xi\left(1+\mu-\zeta_{2}^{2}\right)^{\frac{1}{2}}+v^{\|, \pitchfork} \\
& \dot{\xi}=2 \xi \mu\left(1+\xi-\zeta_{1}^{2}\right)^{\frac{1}{2}} .
\end{aligned}
$$

when $\xi=0$ the $\mu$-subsystem is $\dot{\mu}=v^{\|, \pitchfork}$ which is linear and controllable as desired.

\section{Control design for a nested set stabilization problem}

We now illustrate how these results can be used to locally solve certain set stabilization problems. The following notation is used in this section. Given a nonempty set $A \subset \mathbb{R}^{n}$, point $x \in \mathbb{R}^{n}$, and a vector norm $\|\cdot\|: \mathbb{R}^{n} \rightarrow \mathbb{R}$, the point-to-set distance is defined as $\operatorname{dist}(x, A):=\inf \{\|x-y\|: y \in A\}$. We denote by $\mathcal{N}(A)$ an open neighbourhood of $A$. Given $\epsilon>0$ then $B_{\epsilon}(A):=$ $\left\{x \in \mathbb{R}^{n}: \operatorname{dist}(x, A)<\epsilon\right\}$. By $\phi\left(t, x_{0}\right)$ we denote the solution of (1) with $u=0$ and initial condition $x_{0}$. Let $\phi(t, A):=\left\{\phi\left(t, x_{0}\right): x_{0} \in A\right\}$

The normal form (3) finds application in the stabilization of $S_{2}$ relative to $S_{1}$ locally. For, if $v^{\|, \pitchfork}$ is designed to stabilize $\mu=0$ and the trajectories of the closed-loop system are bounded, then the controller locally stabilizes $S_{2}$ relative to $S_{1}$ in original coordinates. If, on the other hand, the trajectories of the closed-loop system are not bounded, then the stabilization of $\mu=0$ implies the stabilization of $S_{2}$ relative to $S_{1}$ if and only if the necessary and sufficient conditions of [9, Theorem IV.1] hold. Similarly, the refined normal form (23) can simplify the problem of designing controllers to locally stabilize $S_{1}$. If $v^{\pitchfork}$ is designed such that $\xi=0$ is asymptotically stable and the trajectories of the closed-loop system are bounded, then the controller renders $S_{1} \cap U$ asymptotically stable. If, on the other hand, the trajectories of the closed-loop system are not all bounded, then the stabilization of $\xi=0$ implies the local stabilization of $S_{1}$ under necessary and sufficient conditions of [9, Theorem IV.1]. We now present a local solution to a nested set stabilization problem using the results of Section 4. For the reader's convenience we first provide definitions of relative set stability. 


\section{Definition 7 ([10])}

1. The set $S_{2}$ is stable relative to $S_{1}$ for the dynamical system $\dot{x}=f(x)$ if, for all $\epsilon>0$ there exists a neighbourhood $\mathcal{N}\left(S_{2}\right)$ such that for all $t \geq 0, \phi\left(t, \mathcal{N}\left(S_{2}\right) \cap S_{1}\right) \subset B_{\epsilon}\left(S_{2}\right) \cap S_{1}$.

2. The set $S_{2}$ is an attractor relative to $S_{1}$ for the dynamical system $\dot{x}=f(x)$ if there exists a neighbourhood $\mathcal{N}\left(S_{2}\right)$ such that $\operatorname{dist}\left(\phi\left(t, x_{0}\right), S_{2}\right) \rightarrow 0$ as $t \rightarrow \infty$ for all $x_{0} \in \mathcal{N}\left(S_{2}\right) \cap S_{1}$

3. The set $S_{2}$ is asymptotically stable relative to $S_{1}$ for (1) if it is stable and attractive relative to $S_{1}$ for the dynamical system $\dot{x}=f(x)$.

Problem 4. (Local nested set stabilization problem). Given the control system (1), two nested sets $S_{1} \supset S_{2}$ satisfying Assumption 1, a point $\bar{x} \in S_{2}$, and an open set $U$ containing $\bar{x}$ such that $S_{1} \cap U$ and $S_{2} \cap U$ are controlled invariant, find, if possible, a smooth feedback control law such that the closedloop system meets the following three specifications.

S1 The set $S_{1} \cap U$ is asymptotically stable.

S2 The set $S_{2} \cap U$ is asymptotically stable relative to $S_{1} \cap U$.

S3 The set $S_{2} \cap U$ is asymptotically stable.

Notice the requirement that $S_{1} \cap U$ and $S_{2} \cap U$ be controlled invariant is based on the fact that invariance of a set is a necessary condition for its stability [2, Theorem 1.6.6]

\subsection{Stabilizing $S_{1}$}

When Problem 1, 2 or 3 is solvable, the submanifold $S_{1} \cap U$ in transformed coordinates is $\Xi\left(S_{1} \cap U\right)=\{(\zeta, \mu, \xi): \xi=0\}$. It may be possible to utilize normal form (3) or (7) to design a feedback law $v^{\dagger}(\zeta, \mu, \xi)$ that stabilizes the origin of the $\xi$-subsystem. However, if it happens that all the conditions of Theorem 3 hold one can solve Problem 3 which has a decoupled, linear and controllable $\xi$-subsystem. Thus, designing $v^{\text {h }}$ is considerably simplified. We select the simplest controller

$$
v^{\pitchfork}=K_{1} \xi
$$

with $K_{1} \in \mathbb{R}^{\sigma \times n-s_{1}}$ such that $E+F K_{1}$ is Hurwitz. For fast convergence to $S_{1}$ one typically chooses the matrix $K_{1}$ so that the eigenvalues of $E+F K_{1}$ are far in the open left-half complex plane. With the above choice the origin of the $\xi$-subsystem in (23) is rendered exponentially stable and under necessary and sufficient conditions of [9, Theorem IV.1] $\xi \rightarrow 0$ if and only if $x \rightarrow S_{1} \cap U$. 
6.2 Stabilizing $S_{2}$ relative to $S_{1}$

In the normal forms of both Problems 1 and 3 the dynamics restricted to $\Xi\left(S_{1} \cap U\right)$ are

$$
\begin{aligned}
& \dot{\zeta}=f_{1}(\zeta, \mu, 0)+g_{11}(\zeta, \mu, 0) v^{\|}+g_{12}(\zeta, \mu, 0) v^{\|, \pitchfork} \\
& \dot{\mu}=A \mu+B v^{\|, \pitchfork} .
\end{aligned}
$$

Since $(A, B)$ is controllable, there exists a linear feedback

$$
v^{\|, \pitchfork}=K_{2} \mu
$$

with $K_{2} \in \mathbb{R}^{\rho \times s_{1}-s_{2}}$, such that $A+B K_{2}$ is Hurwitz; thus control law (29) exponentially stabilizes the origin of the $\mu$-subsystem restricted to $\xi\left(S_{1} \cap U\right)$ and under necessary and sufficient conditions of [9, Theorem IV.1], $\mu \rightarrow 0$ if and only if $x \rightarrow S_{2} \cap U$

It should be noted that the $\zeta$-subsystem describes the dynamics tangent to both $S_{1}$ and $S_{2}$. When restricted to $S_{2}$, control system (1) evolves according to (6). In some cases it may be possible to utilize the remaining control inputs $v^{\|}$to control dynamics (6) to accomplish application specific specifications such as boundedness or tracking.

\subsection{Stability analysis}

When Problem 3 is solvable the following theorem presents sufficient conditions under which Problem 4 is solvable. Given a continuous signal $u$ let $\|u\|_{\infty}:=$ $\sup _{t>0}\|u(t)\|$ where the norm on the left is a function norm and the norm on the right is a vector norm.

Theorem 4 Assume Problem 3 is solvable at $\bar{x} \in S_{2}$. If

(a) the feedback laws for $v^{\pitchfork}$ and $v^{\|, \pitchfork}$ are given by (27) and (29), respectively.

(b) The control signal $v^{\|}$is such that

(i) $(\forall x(0) \in U)(\forall t \geq 0) x(t) \in U$.

(ii) $(\forall x(0) \in U)(\exists M>0)\|(\zeta, \mu, \xi)\|_{\infty}<M$.

Then Problem 4 is solvable.

Proof. Since Problem 3 is solvable there exists a neighbourhood $U$, a coordinate transformation $\Xi \in \operatorname{Diff}(U)$, and a feedback transformation $(\alpha, \beta)$ such that (1) is locally feedback equivalent to (23). By (a) the closed-loop system is given by

$$
\begin{aligned}
\dot{\zeta} & =\tilde{f}_{1}(\zeta, \mu, \xi)+g_{11}(\zeta, \mu, \xi) v^{\|} \\
\dot{\mu} & =\left(A+B K_{2}\right) \mu+\tilde{f}_{2}(\zeta, \mu, \xi)+g_{21}(\zeta, \mu, \xi) v^{\|} \\
\dot{\xi} & =\left(E+F K_{1}\right) \xi
\end{aligned}
$$

where $\tilde{f}_{1}(\zeta, \mu, \xi):=f_{1}(\zeta, \mu, \xi)+g_{12}(\zeta, \mu, \xi) K_{2} \mu+g_{13}(\zeta, \mu, \xi) K_{1} \xi$ and $\tilde{f}_{2}=$ $f_{2}(\zeta, \mu, \xi)+g_{22}(\zeta, \mu, \xi) K_{2} \mu+g_{23}(\zeta, \mu, \xi) K_{1} \xi$. By conditions (a) and (b), if 
$x(0) \in U$, then (1) is feedback equivalent to (30) for all $t \geq 0$ and $(\zeta, \mu, \xi)$ is bounded.

Let $V_{\zeta} \times V_{\mu} \times V_{\xi}:=\Xi(U)$. The $\xi$-subsystem is decoupled from the other subsystems and $\xi=0$ is exponentially stable by (a). Therefore, by (b), for any $x(0) \in U, \xi \rightarrow 0$. In particular

1. $\left(\forall \epsilon_{1}>0\right)\left(\exists \delta_{1}>0\right)\left(\forall \xi(0) \in B_{\delta_{1}}(0)(\forall t \geq 0) \xi(t) \in B_{\epsilon_{1}}(0)\right.$.

2. $\xi(t) \rightarrow 0$ as $t \rightarrow \infty$ at an exponential rate.

By continuity $\Xi^{-1}\left(V_{\zeta} \times V_{\mu} \times B_{\epsilon_{1}}\right)=: \mathcal{N}_{1}\left(S_{1} \cap U\right)$ and $\Xi^{-1}\left(V_{\zeta} \times V_{\mu} \times B_{\delta_{1}}\right)=$ : $\mathcal{N}_{2}\left(S_{1} \cap U\right)$ are neighbourhoods of $S_{1} \cap U$. Thus, we conclude that

$$
\left(\forall x(0) \in \mathcal{N}_{2}\left(S_{1} \cap U\right)\right)(\forall t \geq 0) x(t) \in \mathcal{N}_{1}\left(S_{1} \cap U\right)
$$

which means that $S_{1} \cap U$ is stable. Moreover, since $\xi(t)$ is bounded, $\xi \rightarrow 0$ if and only if $x \rightarrow S_{1} \cap U$ which implies that $S_{1} \cap U$ is also attractive for all $x_{0} \in U$. Thus, $S_{1} \cap U$ is asymptotically stable and $\mathbf{S} 1$ of Problem 4 holds.

When $\xi=0$, the $\mu$-subsystem becomes $\dot{\mu}=\left(A+B K_{2}\right) \mu$ and $\mu=0$ is exponentially stable, by (a), for all initial conditions in $V_{\zeta} \times V_{\mu} \times\{0\}$. Thus, when $\xi=0$

1. $\left(\forall \epsilon_{2}>0\right)\left(\exists \delta_{2}>0\right)\left(\forall \mu(0) \in B_{\delta_{2}}(0)\right)(\forall t \geq 0) \mu(t) \in B_{\epsilon_{2}}(0)$.

2. $\mu(t) \rightarrow 0$ as $t \rightarrow \infty$ at an exponential rate.

By continuity of $\Xi$ restricted to $S_{1} \cap U$ the sets $\Xi^{-1}\left(V_{\zeta} \times B_{\epsilon_{2}} \times\{0\}\right)=$ : $\mathcal{N}_{3}\left(S_{2} \cap U\right), \Xi^{-1}\left(V_{\zeta} \times B_{\delta_{2}} \times\{0\}\right)=: \mathcal{N}_{4}\left(S_{2} \cap U\right)$ are open neighbourhoods of $S_{2} \cap U$ in the topology of $S_{1} \cap U$. We emphasize that $\mathcal{N}_{3}, \mathcal{N}_{4} \subseteq S_{1} \cap U$. These considerations yield

$$
\left(\forall x(0) \in \mathcal{N}_{4}\left(S_{2} \cap U\right)\right)(\forall t \geq 0) x(t) \in \mathcal{N}_{3}\left(S_{2} \cap U\right)
$$

which means that $S_{2} \cap U$ is stable relative to $S_{1} \cap U$. Moreover, since $\mu(t)$ is bounded, $\mu \rightarrow 0$ if and only if $\left.x\right|_{S_{1} \cap U} \rightarrow S_{2} \cap U$ which implies that $S_{2} \cap U$ is attractive relative to $S_{1} \cap U$. Thus $S_{2} \cap U$ is asymptotically stable relative to $S_{1} \cap U$ and $\mathbf{S 2}$ of Problem 4 holds.

Since : (1) $\xi=0$ is exponentially stable, (2) when $\xi=0, \mu=0$ is exponentially stable and (3) by hypothesis (b) $(\mu(t), \xi(t))$ is bounded all the conditions of [15, Corollary 10.3.3] hold. Therefore $(\mu, \xi)=(0,0)$ is asymptotically stable. That is

1. $\left(\forall \epsilon_{3}>0\right)\left(\exists \delta_{3}>0\right)\left(\forall(\mu(0), \xi(0)) \in B_{\delta_{3}}(0)\right)(\forall t \geq 0)(\mu(t), \xi(t)) \in B_{\epsilon_{3}}(0)$.

2. $(\mu(t), \xi(t)) \rightarrow 0$ as $t \rightarrow \infty$.

By continuity $\Xi^{-1}\left(V_{\zeta} \times B_{\epsilon_{3}}\right)=: \mathcal{N}_{5}\left(S_{2} \cap U\right)$ and $\Xi^{-1}\left(V_{\zeta} \times B_{\delta_{3}}\right)=: \mathcal{N}_{6}\left(S_{2} \cap U\right)$ are neighbourhoods of $S_{2} \cap U$ in $U \subseteq \mathbb{R}^{n}$. Therefore

$$
\left(\forall x(0) \in \mathcal{N}_{6}\left(S_{2} \cap U\right)\right)(\forall t \geq 0) x(t) \in \mathcal{N}_{5}\left(S_{2} \cap U\right)
$$

which means that $S_{2} \cap U$ is stable. Moreover, since $(\mu(t), \xi(t))$ is bounded we have that $(\mu, \xi) \rightarrow 0$ if and only if $x \rightarrow S_{2} \cap U$ which implies that $S_{2} \cap U$ is also attractive for all $x_{0} \in U$. Thus, $S_{2} \cap U$ is asymptotically stable and $\mathbf{S 3}$ of Problem 4 holds. 


\subsection{Example}

For illustration purposes we consider the control system

$$
\dot{x}=\left[\begin{array}{c}
-x_{2} \\
x_{1} \\
x_{1} x_{3}
\end{array}\right]+\left[\begin{array}{c}
x_{1} \\
x_{2} \\
1
\end{array}\right] u_{1}+\left[\begin{array}{c}
-x_{2} \\
x_{1} \\
x_{1}^{2}+x_{2}^{2}
\end{array}\right] u_{2}
$$

and sets

$$
S_{1}=\left\{x \in \mathbb{R}^{3}: x_{1}^{2}+x_{2}^{2}-1=0\right\}, S_{2}=\left\{x \in \mathbb{R}^{3}: x_{1}^{2}+x_{2}^{2}-1=x_{3}=0\right\}
$$

One can verify that the conditions of Theorem 3 hold, thus Problem 3 is solvable. Following the proof of Theorem 3 we find the following feedback transformation and coordinate transformation

$$
(\alpha, \beta)=\left(\left[\begin{array}{c}
0 \\
-x_{1} x_{3} \\
x_{1}^{2}+x_{2}^{2}
\end{array}\right],\left[\begin{array}{cc}
0 & \frac{1}{2\left(x_{1}^{2}+x_{2}^{2}\right)} \\
\frac{1}{x_{1}^{2}+x_{2}^{2}} & 0
\end{array}\right]\right), \quad \Xi=\left[\begin{array}{c}
x_{2} \\
x_{3} \\
x_{1}^{2}+x_{2}^{2}-1
\end{array}\right]
$$

The given control system is feedback equivalent to

$$
\begin{aligned}
& \dot{\zeta}=-\left(1+\xi-\zeta^{2}\right)^{\frac{1}{2}}-\frac{\left(1+\xi-\zeta^{2}\right) \mu}{\xi+1}-\frac{\left(1+\xi-\zeta^{2}\right)^{\frac{1}{2}}}{\xi+1} v^{\|, \pitchfork}+\frac{\zeta}{2(\xi+1)} v^{\pitchfork} \\
& \dot{\mu}=v^{\|, \pitchfork}+\frac{1}{2(\xi+1)} v^{\pitchfork} \\
& \dot{\xi}=v^{\pitchfork} .
\end{aligned}
$$

We design $v^{\pitchfork}=k_{1} \xi$ and $v^{\|, \pitchfork}=k_{1} \mu$ with $k_{1}=-1$ and $k_{2}=-1$. Figure $1(\mathrm{~b})$ illustrates the trajectories of the closed-loop system and Figure 1(a) shows the projected trajectories to $\left(x_{1}, x_{2}\right)$-plane. The set stabilization error for $S_{1}$ is

$$
\operatorname{dist}\left(x, S_{1}\right):=\sqrt{x_{1}^{2}+x_{2}^{2}-1}
$$

and for $S_{2}$ is

$$
\operatorname{dist}\left(x, S_{2}\right):=\sqrt{x_{3}^{2}+\sqrt{x_{1}^{2}+x_{2}^{2}-1}}
$$

Figures 2(a) and 2(b) display $\operatorname{dist}\left(x, S_{1}\right)$ and $\operatorname{dist}\left(x, S_{2}\right)$, receptively. 

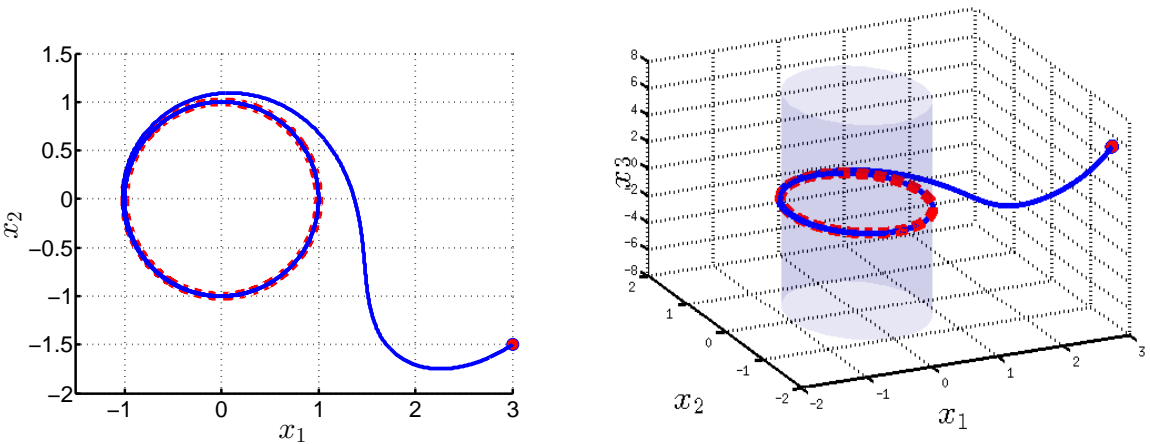

(a) trajectories of the closed-loop system in (b) trajectories of the closed-loop system in $\left(x_{1}, x_{2}\right)$-plane $\mathbb{R}^{3}$

Fig. 1: Stabilization of $S_{1}$ and $S_{2}$

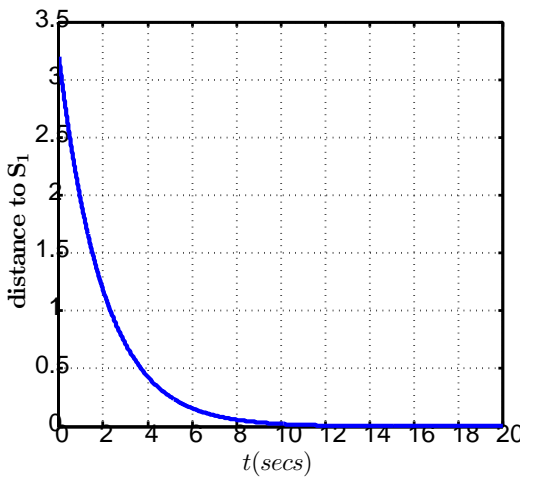

(a) distance to $S_{1}$

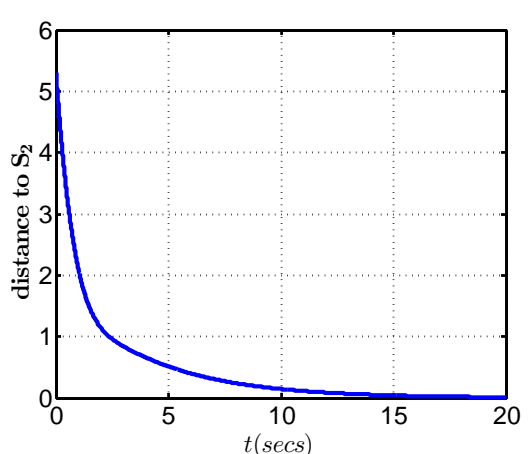

(b) distance to $S_{2}$

Fig. 2: Set stabilization errors $S_{1} \supset S_{2}$

\section{Conclusions}

We have determined necessary and sufficient conditions under which a nonlinear control-affine system, with two nested, controlled invariant, embedded submanifolds in its state space, is feedback equivalent to a system whose dynamics restricted to the larger submanifold are locally transversally feedback linearizable with respect to the nested set. We called this Problem 1. When Problem 1 is solvable we have presented further necessary and sufficient condi- 
tions under which Problem 3 is solvable where it is additionally asked for the dynamics transversal the the larger set to be linear and controllable as well. Sufficient conditions are presented under which one can utilize these normal forms to solve a nested set stabilization problem locally.

A global version of Problems 1, 2, and 3 can be posed. Roughly speaking, one seeks a coordinate and feedback transformation such that (1) is feedback equivalent to (3), in Problem 1, to (7), in Problem 2, and to (23), in Problem 3, in a tubular neighbourhood of the nested set. Accordingly, the global version of Problem 3 can be employed to solve the global version of the nested set stabilization problem where the entire sets are considered.

\section{Appendix A Supporting results and proofs}

Proof of Proposition 1. The proof that $\operatorname{dim}(P(q))=\nu(q)$ is obvious from their definitions and is omitted. Next we have

$$
\begin{aligned}
& \operatorname{dim}(Q(q)) \\
& =n-\operatorname{dim}\left(G_{0}(q) \cap T_{q} S_{2}\right)+\operatorname{dim}\left(G_{0}(q) \cap T_{q} S_{1}\right)-\operatorname{dim}\left(\left[G_{0}(q) \cap T_{q} S_{2}\right]^{\perp}+\left[G_{0}(q) \cap T_{q} S_{1}\right]\right) \\
& =\operatorname{dim}\left(G_{0}(q) \cap T_{q} S_{1}\right)-\operatorname{dim}\left(G_{0}(q) \cap T_{q} S_{2}\right) \\
& =\rho(q) .
\end{aligned}
$$

Similar computations yield $\operatorname{dim}(R(p))=\sigma(p)$ on $S_{1}$.

Proof of Proposition 2. Let $U \subseteq \mathbb{R}^{n}$ be an open set containing $\bar{x}$ and set $V_{1}=$ $S_{1} \cap U$. If $\operatorname{dim}\left(T_{x} S_{1} \cap G_{0}(x)\right)$ is constant on $V_{1}$ then, since $\operatorname{dim}\left(T_{x} S_{1}\right)$ and $\operatorname{dim}\left(G_{0}(x)\right)$ are constant on $V_{1}$, the function $\sigma(x)$ in (11) is constant on $V_{1}$. If both $\operatorname{dim}\left(T_{x} S_{2} \cap G_{0}(x)\right)$ and $\operatorname{dim}\left(T_{x} S_{1} \cap G_{0}(x)\right)$ are constant on $V_{2}=S_{2} \cap U$ then the functions $\nu$ and $\rho$ in (11) are constant on $V_{2}$.

Conversely, if the function $\sigma$ is constant on an open set $V_{1} \subset S_{1}$ with $\bar{x} \in V_{1}$, then since $T_{x} S_{1}$ and $G_{0}(x)$ are constant dimensional and from the definition of $\sigma$ it follows that $\operatorname{dim}\left(T_{x} S_{1} \cap G_{0}(x)\right)$ is constant on $V_{1}$. If $\nu, \rho$ are constant on an open set $V_{2} \subset S_{2}$ with $\bar{x} \in V_{2}$ then from their definitions it follows that $\operatorname{dim}\left(T_{x} S_{2} \cap G_{0}(x)\right)$ is constant on $V_{2}$.

Proof of Proposition 3. Let $\bar{x} \in S_{2}$ be a regular point of the distributions (10). Then by Proposition 2 and Definition $4 P$ is non-singular in a neighbourhood $V_{2}=V_{1} \cap S_{2}$, with $V_{1} \subseteq S_{1}$ and containing $\bar{x}$. Lemma A1 proves that $P$ is also smooth in a neighbourhood of $\bar{x}$, without loss of generality, $V_{2}$. Proposition 1 shows that $Q$ is non-singular on $V_{2}$ and $R$ is non-singular on $V_{1}$. Furthermore, by Proposition 2, the assumed non-singularity of $G_{0}$ and Lemma A1 we have, by possibly shrinking $V_{1}$, and hence $V_{2}$, that $G_{0} \cap T S_{1}$ and $\left[G_{0} \cap T S_{1}\right]^{\perp}$ are smooth on $V_{1}$ and $\left[G_{0}(x) \cap T S_{2}\right]^{\perp}$ is smooth on $V_{2}$. Therefore $Q$ and $R$ are the non-singular intersection of smooth non-singular distributions and by [16, Lemma 1.3.5] they are smooth themselves. 
Conversely, suppose that the distribution $R$ in (10) is smooth and nonsingular in a neighbourhood $V_{1} \subseteq S_{1}$ containing $\bar{x}$ and distributions $P$ and $Q$ in (10) are smooth and non-singular in $V_{2}=V_{1} \cap S_{2}$. By Proposition 1 and Definition $4 \bar{x}$ is a regular point of (11).

Proof of Lemma 1. Let $x \in S_{2}$ be fixed but arbitrary and let $\Xi \in \operatorname{Diff}(U)$ be a diffeomorphism onto its image with domain $U$ containing $x$. Let $(\alpha, \beta)$ be a regular feedback transformation also defined on $U$ and let $\tilde{g}(x):=g(x) \beta(x)$, $\tilde{G}_{0}(x):=\operatorname{span}\left\{\tilde{g}_{1}(x), \cdots, \tilde{g}_{m}(x)\right\}$. Since each $\tilde{g}_{i}(x)$ is a linear combination of $g_{1}(x), \ldots, g_{m}(x), \tilde{G}_{0}(x) \subseteq G_{0}(x)$. Furthermore, since $\beta: U \subseteq \mathbb{R}^{n} \rightarrow \mathrm{GL}(m, \mathbb{R})$ is non-singular, $\tilde{G}_{0}(x)=G_{0}(x)$ and therefore

$$
\nu(x)=\operatorname{dim}\left(T_{x} S_{2} \cap G_{0}(x)\right)=\operatorname{dim}\left(T_{x} S_{2} \cap \tilde{G}_{0}(x)\right) .
$$

Next, let $\hat{g}:=\Xi_{\star}(g \beta)=\Xi_{\star}(\tilde{g})$ and $\hat{G}_{0}:=\operatorname{span}\left\{\hat{g}_{1}, \cdots, \hat{g}_{m}\right\}$. Since $\mathrm{d} \Xi_{x}$ is an isomorphism at each $x \in U$, we have

$$
\begin{aligned}
\operatorname{dim}\left(T_{x} S_{2} \cap \tilde{G}_{0}(x)\right) & =\operatorname{dim}\left(\mathrm{d} \Xi_{x}\left(T_{x} S_{2} \cap \tilde{G}_{0}(x)\right)\right)=\operatorname{dim}\left(\mathrm{d} \Xi_{x}\left(T_{x} S_{2}\right) \cap \mathrm{d} \Xi_{x}\left(\tilde{G}_{0}(x)\right)\right) \\
& =\operatorname{dim}\left(T_{\Xi(x)} \Xi\left(S_{2} \cap U\right) \cap \hat{G}_{0}(\Xi(x))\right)
\end{aligned}
$$

where the next to last equality comes from the fact that $\operatorname{Ker}\left(\mathrm{d} \Xi_{x}\right)=\{0\}$. From this it follows that the value $\nu(x)$ is unchanged under coordinate and feedback transformations. The same arguments hold for the other functions in (11).

Lemma A1 ([29]). Let $N \subset M$ be an $n$-dimensional submanifold of the $m$ dimensional manifold $M$. Let $p \in N$ be a regular point of a d-dimensional distribution $D$ on $M$. Suppose there exists an open neighbourhood $V$ of $p$ in $N$ such that $k=\operatorname{dim}\left(T_{q} N \cap D(q)\right)$ is constant for all $q \in V$. Then, there exists a neighbourhood $U$ of $p$ in $V$ such that $T N \cap D$ is smooth on $U$.

Proof. Let $(W, \psi)$ be a coordinate chart of $M$ adapted to $N$, that is, such that $\psi(N \cap W)=\left\{x \in \psi(W): x_{n+1}=\cdots=x_{m}=0\right\}$, and let $\left\{f_{1}, \ldots, f_{d}\right\}$ be a set of local generators of $D$ around $p$. Let $\pi:\left(x_{1}, \ldots, x_{m}\right) \mapsto\left(x_{1}, \ldots, x_{n}\right)$ be the projection onto the first $n$ factors. By making $W$ smaller, we can assume that $f_{1}, \ldots, f_{d}$ are linearly independent on $W$.

Recall that $\hat{\psi}:=\pi \circ \psi: N \cap W \rightarrow \mathbb{R}^{n}$ is a diffeomorphism onto its image, and let

$$
\hat{f}_{i}:=\hat{\psi}_{\star}\left(f_{i}\right), i \in\{1, \ldots, d\} .
$$

The vector fields $\hat{f}_{i}$ are defined on an open set of $\mathbb{R}^{n}$. Letting $\left\{e_{1}, \ldots, e_{n}\right\}$ denote the natural basis of $\mathbb{R}^{n}$, for each $q \in N \cap W$ we have

$$
\begin{aligned}
d \hat{\psi}_{q}\left(T_{q} N \cap D(q)\right) & =d \hat{\psi}_{q}\left(T_{q} N\right) \cap d \hat{\psi}_{q}(D(q)) \\
& =\operatorname{span}\left\{e_{1}, \ldots, e_{n}\right\} \cap \operatorname{span}\left\{\hat{f}_{1}(\psi(q)), \ldots, \hat{f}_{d}(\psi(q))\right\} .
\end{aligned}
$$

Hence, $d \hat{\psi}(T N \cap D)$ is a distribution on an open set of $\mathbb{R}^{n}$. By assumption, and since $d \hat{\psi}_{q}$ is an isomorphism at each $q \in N \cap W$, it is the intersection 
of two smooth non-singular distributions, and it has constant dimension near $\psi(p)$. Therefore, by [16, Lemma 1.3.5], it is smooth. This implies that $T N \cap D$ is also smooth on a neighbourhood $V$ of $p$.

\section{Compliance with ethical standards}

This research was partially supported by the Natural Sciences and Engineering Research Council of Canada (N.S.E.R.C.). Alireza Doosthoseini is a Ph.D. candidate at University of Waterloo and Christopher Nielsen is an associate professor at University of Waterloo.

\section{References}

1. Banaszuk A, Hauser J (1995) Feedback linearization of transverse dynamics for periodic orbits. Systems \& Control Letters 26:95-105

2. Bhatia NP, Szegö GP (1967) Dynamical Systems : Stability theory and applications. Springer-Verlag, Berlin

3. Brockett RW (1978) Feedback invariants for nonlinear systems. In: Proceedings of the IFAC World Congress, Helsinki, pp 1115-1120

4. Cartan E (1914) Sur l'quivalence absolue de certains systmes d'quations diffrentielles et sur certaines familles de courbes. Bulletin de la Socit Mathmatique de France 42:12-48

5. Consolini L, Maggiore M, Nielsen C, Tosques M (2010) Path following for the PVTOL aircraft. Automatica 46(8):1284-1296

6. Doosthoseini A, Nielsen C (2013) Coordinated path following for a multiagent system of unicycles. In: Proceedings of the 52nd Conference on Decision and Control, IEEE, Florence, pp 2894-2899

7. Dörfler F, Bullov F (2014) Synchronization in complex networks of phase oscillators: A survey. Automatica 50(6):1539-1564

8. El-Hawwary M, Maggiore M (2010) Reduction principles and the stabilization of closed sets for passive systems. IEEE Transactions on Automatic Control 55(4):982-987

9. El-Hawwary MI, Maggiore M (2007) Passivity-based stabilization of noncompact sets. In: Proceedings of the 46th IEEE Conference on Decision and Control, New Orleans, USA

10. El-Hawwary MI, Maggiore M (2013) Reduction theorems for stability of closed sets with application to backstepping control design. Automatica 49(1):214-222

11. Gardner R, Shadwick W (1987) Feedback equivalence of control systems. Systems \& Control Letters 8(5):463-465

12. Gardner R, Shadwick W (1990) Feedback equivalence for general control systems. Systems \& Control Letters 15(1):15-23

13. Guay M (1999) An algorithm for orbital feedback linearization of singleinput control affine systems. Systems \& Control Letters 38(4):271-281 
14. Hermann R (1988) Invariants for feedback equivalence and cauchy characteristic multifoliations of nonlinear control systems. Acta Applicandae Mathematica 11(2):123-153

15. Isidori A (1887) Nonlinear control systems., vol 2. Springer

16. Isidori A (1995) Nonlinear control systems., vol 1, 3rd edn. Springer

17. Isidori A, Krener A (1982) On feedback equivalence of nonlinear systems. Systems \& Control Letters 2(2):118-121

18. Isidori A, Ruberti A (1984) On the synthesis of linear input-output responses for nonlinear systems. Systems \& Control Letters 4(1):17-22

19. Kang W (1994) Approximate linearization of nonlinear control systems. Systems \& Control Letters 23(1):43-52

20. Krener AJ (1973) On the equivalence of control systems and the linearization of nonlinear systems. SIAM Journal on Control and Optimization 11(4):670-676

21. Krener AJ (1984) Approximate linearization by state feedback and coordinate change. Systems \& Control Letters 5(3):181-185

22. Krener AJ, Maag B (1991) Controller and observer design for cubic systems. In: Modeling, Estimation and Control of Systems with Uncertainty, Springer, pp 224-239

23. Krener AJ, Isidori A, Respondek W (1983) Partial and robust linearization by feedback. In: Proceedings of the 22nd IEEE Conference on Decision and Control, San Antonio, pp 126-130

24. Krener AJ, Karahan S, Hubbard M, Frezza R (1987) Higher order linear approximations to nonlinear control systems. In: Proceedings of the 26th IEEE Conference on Decision and Control, IEEE, Los Angeles, vol 26, pp $519-523$

25. Krener AJ, Karahan S, Hubbard M (1988) Approximate normal forms of nonlinear systems. In: Proceedings of the 27th IEEE Conference on Decision and Control, IEEE, Austin, pp 1223-1229

26. Lee JM (2003) Introduction to smooth manifolds, volume 218 of graduate texts in mathematics. Springer-Verlag, New York

27. Marino R (1986) On the largest feedback linearizable subsystem. Systems \& Control Letters 6(5):345-351

28. Marino R, Boothby W, Elliott DL (1985) Geometric properties of linearizable control systems. Mathematical Systems Theory 18(1):97-123

29. Nielsen C (2009) Set stabilization using transverse feedback linearization. PhD thesis, University of Toronto

30. Nielsen C, Maggiore M (2008) On local transverse feedback linearization. SIAM Journal on Control and Optimization 47(5):2227-2250

31. Olfati-Saber R, Murray RM (2004) Consensus problems in networks of agents with switching topology and time-delays. IEEE Transactions on Automatic Control 49(9):1520-1533

32. Poincaré H (1879) Sur les propriétés des fonctions définies par les équations aux différences partielles. 4, Gauthier-Villars, Paris

33. Respondek W, Tall I (2006) Feedback equivalence of nonlinear control systems: a survey on formal approach. Chaos in Automatic Control pp 
137-262

34. Shadwick W (1990) Absolute equivalence and dynamic feedback linearization. Systems \& Control Letters 15(1):35-39

35. Sommer R (1980) Control design for multivariable non-linear time-varying systems. International Journal of Control 31(5):883-891

36. Su R (1982) On the linear equivalents of nonlinear systems. Systems \& Control Letters 2(1):48-52

37. Wonham W (2012) Linear Multivariable Control: A Geometric Approach. Stochastic Modelling and Applied Probability, Springer New York 